Ilias Tsiakas 


\title{
Periodic Stochastic Volatility and Fat Tails
}

\author{
Ilias Tsiakas* \\ Warwick Business School, UK
}

April 23, 2004

\begin{abstract}
This paper provides a comprehensive analysis of the day of the week, non-trading day (post-holiday), and month of the year seasonal effects in the daily returns and volatility of the S\&P 500 index. We use bootstrapping to demonstrate that, surprisingly, these three prominent calendar effects are statistically significant in daily volatility, but not in daily average returns. We model this form of seasonal heteroscedasticity by introducing the periodic stochastic volatility $(P S V)$ model for characterizing the seasonal patterns of daily financial market volatility. We analyze the interaction of seasonal heteroscedasticity with fat tails by comparing the performance of Gaussian PSV and fat-tailed PSVt specifications to the plain vanilla $S V$ and $S V t$ benchmarks. Consistent with the bootstrapping results, we find strong evidence of seasonal periodicity in volatility, which substantially reduces the need for fat tails, and is robust to the exclusion of the Crash of 1987 outliers. The $S V$ parameters are estimated by implementing the Bayesian MCMC methods developed by Chib, Nardari and Shephard (2002), with the addition of a Gibbs step for sampling the seasonal volatility level effects. We compute in-sample and outof-sample density forecasts for assessing the adequacy of the conditional distribution. We conclude by using Bayes factors as a likelihood-based framework for ranking the $S V$ specifications.
\end{abstract}

Keywords: Stochastic Volatility, Seasonal Periodicity, Seasonal Heteroscedasticity, Bayesian MCMC Estimation.

JEL Classification: C11, C15, C22, C51, C52, C53, G10.

${ }^{*}$ Accounting and Finance Department, Warwick Business School, University of Warwick, Coventry CV4 7AL, United Kingdom. Tel: +44 (0)2476 524966. Fax: +44 (0)2476 523779. Email: ilias.tsiakas@wbs.ac.uk. I have benefited from the comments of seminar participants at the ISMA Centre, University of Reading, and the 2003 summer meetings of the Econometric Society at Chicago and Stockholm. I am especially grateful to Angelo Melino, Tom McCurdy and Roel Oomen for many helpful comments and discussions. This work was partially completed as a chapter in the author's $\mathrm{PhD}$ dissertation at the University of Toronto. 


\section{Introduction}

It is widely documented that expected stock returns exhibit strong seasonal patterns in the form of day of the week, month of the year and post-holiday effects (Lakonishok and Smidt (1988)). For example, Keim (1983) and Reinganum (1983) showed that small firms display abnormal returns (measured relative to the CAPM) during the first two weeks of January. This anomaly became known as the "turn-of-the-year effect", and is more frequently referred to as the "January effect". French (1980) and Keim and Stambaugh (1984) observed that the average return to the $\mathrm{S} \& \mathrm{P}$ composite portfolio over weekends was negative and statistically significant. This second calendar anomaly is known as the "weekend effect". Further, French and Roll (1986) and Baillie and Bollerslev (1989) noted that volatility tends to be higher following non-trading days (post-holidays). We refer to this as the "holiday effect" or "nontrading day" effect.

Subsequent to their discovery, there have been many attempts to justify these seasonalities $^{1}$. However, there is still no equilibrium model of asset prices, which can explain why expected returns should display calendar regularities. Possible explanations include inventory adjustments of different traders (Ritter (1988)), the timing of trades by informed and uninformed traders (Admanti and Pfeiderer (1988)), specialists' strategies in response to informed traders (Admanti and Pfeiderer (1989)), the timing of corporate news releases (Penman (1987)), tax induced trading (Lakonishok and Smidt (1986)), and the window dressing induced by periodic evaluation of portfolio managers (Haugen and Lakonishok (1988)). Other theories focus on the institutional arrangements in financial markets. For example, the January effect has been linked to year-end tax-loss selling pressure, which reduces stock prices in December, leading to a rebound in early January as investors repurchase these stocks to reestablish investment positions (Roll (1983)). Glosten, Jagannathan and Runkle (1993) also suggest that consumer sales exhibit a pronounced quarterly seasonal pattern, of which the fourth quarter is the important holiday season. January is the period when comprehensive and reliable information about consumer spending typically becomes available. Explanations offered for the strong Monday effect in stock returns include delays between trading and settlements in stocks (Lakonishok and Levi (1982)), measurement error (Keim and Stambaugh (1984)), institutional factors (Flannery and Protopapadakis (1988)), and trading patterns (Lakonishok and Maberly (1990)).

This paper provides a comprehensive analysis of the day of the week, non-trading day, and month of the year seasonal effects in the daily returns and, more importantly, in the volatility of the S\&P 500 index over the years 1980-2001. Specifically, we find that Mondays tend to have the lowest return and highest volatility. Wednesdays have the second highest return and the lowest volatility. January has the highest return, while October has the highest volatility. Finally, the first weekday after a non-trading weekday (a post-holiday) has a negative average return and very high volatility.

\footnotetext{
${ }^{1}$ In fact, Sullivan, Timmermann and White (2001) argue that such theories are "after the fact" rationalizations of observed phenomena.
} 
Even though the seasonal patterns of monthly, daily and intraday returns have received much attention in the finance and econometrics literature, there is surprisingly little work done on the seasonality of daily volatility, even within the broadly used GARCH framework. Perhaps the closest to providing a thorough examination of seasonal periodicity in both the returns and the variance of daily S\&P 500 index is Gallant, Rossi and Tauchen (1992) in their investigation of return-volume comovements. Further, Andersen and Bollerslev (1997) and Beltratti and Morana (1999) study the intraday periodicity of asset returns, which takes the form of cycles repeating within the day and week. Bollerslev and Ghysels (1996) propose a periodic GARCH (P-GARCH) model designed to capture the repetitive seasonal time variation in volatility. Glosten, Jagannathan and Runkle (1993) use a GARCH-M model on monthly equity data, which only conditions on January and October effects. Beller and Nofsinger (1998) examine only month of the year volatility effects using monthly data. Balaban, Bayar and Kan (2001), Berument and Kuymaz (2001), and Frances and Paap (2000) examine only the day of the week effect on stock market volatility.

This investigation has four objectives: (i) to measure the size of the seasonal heteroscedasticity observed in the form of day of the week, non-trading day, and month of the year seasonal level effects in both the daily returns and the daily volatility of the S\&P 500 index; (ii) to provide a careful analysis of the statistical significance of seasonality, which will allow us to report the striking new result that most calendar effects are strongly statistically significant in volatility, but not in expected returns; more importantly (iii) to introduce and estimate the periodic stochastic volatility model $(P S V)$, which is designed to exploit the observed seasonal heteroscedasticity; and finally (iv) to formally analyze the interaction between seasonal periodicity and fat tails by assessing the performance of Gaussian and fat-tailed periodic stochastic volatility specifications set against appropriate plain vanilla $S V$ and $S V t$ benchmarks. The second, and especially the third and fourth objectives are novel contributions to the existing literature.

Early work on seasonal abnormalities in expected stock returns has in general found overwhelming evidence of statistical significance for most calendar effects. For example, Lakonishok and Smidt (1989) implement two-sided $t$ tests and joint $F$ tests on ninety years of Dow Jones Industrial Average (DJIA) daily returns and find that the seasonal patterns in the rates of return are small but very statistically significant. Two recent empirical studies offer conflicting conclusions. On the one hand, Hansen and Lunde (2003) design a $\chi^{2}$ test, which exploits the correlation structure of calendar effects. Using stock indices from 10 countries, they find strong evidence that a large universe of calendar effects is statistically significant in return means and standardized return means. On the other hand, Sullivan, Timmermann and White (2001) develop a new model-free bootstrap procedure, which explicitly measures the distortions on statistical inference induced by data mining. They use 100 years of DJIA daily returns in order to analyze whether a particular set of calendar-based trading rules yields a higher (standardized) return than a buy and hold strategy and conclude that calendar effects are no longer statistically significant. Notably, none of these studies models periodicity in volatility. 
In this paper, we use simple bootstrapping to simulate the empirical distribution of the seasonal return means and absolute return means and perform formal hypothesis testing. The null hypotheses are all one-sided and test whether (for example) the returns of January are equal versus higher (or for other calendar effects lower) than the rest of the months. We demonstrate that, surprisingly, the three prominent calendar effects are statistically significant only in daily volatility and not in daily average returns. We also show that our bootstrapping results are not driven by the Crash 1987 outliers. Specifically, all the results are robust to the exclusion of the three most volatile days of October 1987: Monday, October 19, 1987, when the S\&P 500 composite index plunged $22.9 \%$ (the minimum in our sample) on the second highest volume ever recorded (604 million shares); Tuesday, October 20, when the S\&P 500 index rose by $5.2 \%$ on the highest volume ever recorded (608 million shares); and Wednesday, October 21, when the S\&P 500 rose $8.7 \%$ (the maximum in our sample) with the trading of 450 million shares.

The bootstrapping evidence strongly indicates that a volatility model should perform better if it was generalized to account for the observed seasonal periodicity in volatility. In this context, the main contribution of this paper is that it introduces and estimates the periodic stochastic volatility $(P S V)$ model, and then formally examines the interaction between seasonal heteroscedasticity and fat tails. The $P S V$ model generalizes the constants in both the conditional mean and the conditional variance functions in order to account for the day of the week, holiday, and month of the year seasonal level effects. Specifically, the conditional standard deviation of the periodic SV specifications is subject to a periodic seasonal level effect, which is separate from the dynamic SV component. The most comprehensive periodic specification uses a cyclical and parsimonious six term Fourier approximation, which fits the month of the year effect reasonably well and reduces the parameter dimension of the model. ${ }^{2}$

The study adopts a univariate discrete-time SV framework and tests the performance of Gaussian PSV and fat-tailed PSVt specifications against two benchmark SV models: (i) the plain vanilla SV model, which assumes conditionally Gaussian innovations, an autoregressive conditional mean, and a persistent stochastic log-variance process; and (ii) the fat-tailed $S V t$ model, which is designed to capture the excess kurtosis of daily returns by assuming that daily innovations follow a Student- $t$ distribution. In total, we estimate eight SV specifications.

Unlike statistical jump processes specified in returns and volatility, the timing and size of which are estimated ex-post and are ex-ante unforecastable, ${ }^{3}$ the timing of seasonal periodicity is perfectly observed. Conditioning on seasonality adjusts the level of the returns and the volatility process every day so that the persistent stochastic volatility component can better accommodate the shocks that may be specific (for example) to a Monday in October. We analyze the statistical interaction between seasonal periodicity and fat tails and demonstrate that the PSV model reduces and even eliminates the need for a fat-tailed SV specification.

\footnotetext{
${ }^{2}$ For Flexible Fourier Form (FFF) modelling of intraday periodic volatility components see Andersen and Bollerslev (1997), and Beltratti and Morana (1999). For the details of FFFs in general see Gallant (1981).

${ }^{3}$ Johannes, Kumar and Polson (1999) propose a state dependent jump model, where the jump size and the jump intensity are functions of lagged returns and lagged implied volatilities, and find evidence of predictability.
} 
This result is also robust to the Crash of 1987 outliers.

We compute in-sample and out-of-sample density forecasts for assessing the adequacy of the conditional distribution of the SV specifications. We explicitly test whether modelling the distinct behaviour of different days and months results in better one-step ahead volatility and density forecasts. We also compute Bayes factors, which provide a framework for specification diagnostics and model selection over the set of SV models. Bayes factors account for both estimation risk by integrating out parameter uncertainty and for model risk, which arises from the uncertainty over selecting a model specification. More importantly, the Bayes factor diagnostic measures the statistical cost of dimensionality due to the explicit accounting of all seasonal periodic effects in returns and volatility.

Models of stochastic volatility have been used extensively in theoretical option pricing since the contribution of Hull and White (1987) in generalizing the Black-Scholes option pricing scheme. Like GARCH-type models, they are designed to capture the persistent and hence predictable component of daily volatility (for a comparison of GARCH and SV models see Fleming and Kirby (2003)). However, SV has a fundamental difference with GARCH. The assumption of a stochastic second moment introduces an additional source of risk that cannot be perfectly hedged using $t-1$ information. A GARCH specification describes the conditional distribution of returns as being a function of exclusively past information. In contrast, the SV model specifies the joint conditional distribution of both the return and the volatility process. Intuitively, SV allows for the possibility of random (but rather small) contemporaneous volatility shocks due to news events and policy changes. In other words, there may exist unobserved contemporaneous variables that affect the volatility process, which is not possible in GARCH.

Despite their parsimonious structure, intuitive appeal and popularity in theoretical option pricing, SV models have been much less popular than GARCH in empirical applications. This is exclusively due to the difficulties associated with estimating SV models using conventional Classical econometric methods. Specifically, models of discrete-time stochastic volatility cannot be estimated with likelihood-based methods because the likelihood function is not available analytically. ${ }^{4}$ Bayesian estimation offers a substantial computational advantage over any Classical approach because it avoids tackling very difficult, if not intractable, numerical optimization procedures. This has turned the development of fast and efficient Bayesian MCMC algorithms for the estimation of SV models into one of the most promising and challenging tasks of modern time series analysis. ${ }^{5}$

The SV parameters are estimated by implementing the MCMC algorithm of Chib, Nardari and Shephard (2002), which builds on the procedures developed by Kim, Shephard and Chib (1998). ${ }^{6}$ The specification and model selection tests are based on the filtering methods of Pitt

\footnotetext{
${ }^{4}$ In the Classical framework, Sandmann and Koopman (1998) propose a Monte Carlo Likelihood (MCL) method for estimating simple (plain vanilla) SV models. For a Simulated Maximum Likelihood (SML) estimation method of heavy-tailed SV models see Liesenfeld and Jung (2000).

${ }^{5}$ For a general reference on MCMC methods in financial econometrics see Johannes and Polson (2002).

${ }^{6}$ For an alternative reference on Bayesian analysis of SV models see Jacquier, Polson and Rossi (2002).
} 
and Shephard (1999). The marginal likelihood input to the computation of Bayes factors is constructed as in Chib (1995), and Chib and Jeliazkov (2001).

The paper is organized as follows. Section 2 describes the size and statistical significance of seasonal periodicity in the daily S\&P 500 average returns and average absolute returns. Section 3 discusses the plain vanilla Gaussian $S V$ and the fat-tailed $S V t$ models, and then presents in detail three periodic SV specifications which condition on day of the week, postholiday and month of the year information in both the mean and the conditional variance. The SV specifications lead to the testing hypotheses presented in Section 4. A sketch of the MCMC algorithm is offered in Section 5. Section 6 examines the in-sample and out-of-sample conditional dynamics of the SV models and discusses Bayes factors as a diagnostic tool for model selection. Section 7 discusses the results and Section 8 concludes.

\section{S\&P 500 Returns Data}

Let $P_{t}$ denote the daily closing value of the $\mathrm{S} \& \mathrm{P} 500$ Composite Price Index. Then, the continuously compounded per cent returns are constructed as

$$
y_{t}=100 \log \frac{P_{t}}{P_{t-1}}
$$

The information available to the econometrician at time $t$ is $F_{t}=\left\{y_{t}, y_{t-1}, \ldots, y_{1}\right\}$. The sample size of the daily returns is $T=5552$ and covers the 22 year period from January 1, 1980 to December 31, 2001. The source of the data is Datastream.

\subsection{Descriptive Statistics and Seasonality}

Table 1 presents the descriptive statistics of $y_{t},\left|y_{t}\right|$ and $\log \left|y_{t}\right|$. These statistics verify that daily returns exhibit negative skewness, high kurtosis and low short-lived serial correlation. Absolute returns are extremely noisy and persistent: they have high positive skewness, immense kurtosis, and long-lived positive serial correlation. Finally, the log-absolute returns are much closer to normality than the daily $y_{t}$ and $\left|y_{t}\right|$.

Table 2A presents the day of the week seasonal behaviour of the data. Specifically, Mondays exhibit by far the lowest average return (which is essentially zero) and the highest volatility, where the latter is proxied simply by the mean of $\left|y_{t}\right|$. Further, we pay special attention to the $H O L$ days, which we define as the weekdays that follow a non-trading weekday (i.e. $H O L$ is a post-holiday). HOL days remarkably have a negative average return and very high volatility, which is $24.6 \%$ higher than their complement (i.e. the non-HOL days).

Table 2B displays the month of the year seasonality. October is by far the most volatile month in that the sample mean of the October absolute returns is $29.6 \%$ higher than that of the rest 11 months. January has the highest return and second highest volatility. June is the quietest month (least volatile). An important aspect of these data is shown in Table 2C. 
Specifically, these results remain largely unaffected when we exclude the three most volatile days of the sample: Monday, October 19, 1987 (the minimum), to Wednesday, October 21, 1987 (the maximum). Monday and October are still the most volatile of all days and months, respectively. However, October's volatility is now only $19.4 \%$ higher than its complement.

\subsection{Statistical Significance and Bootstrapping}

Even though it is clear that each day and month has a different sample mean for the daily return and volatility level, it is also crucial to determine which of the seasonal effects are statistically significant. We use bootstrapping to simulate the empirical distribution of the seasonal sample means and perform formal hypothesis testing. This is a first step in determining the significance of periodic seasonality in daily volatility (seasonal heteroscedasticity) by conducting a careful model-free examination of the data. As we will see, the results offer ample motivation for the periodic specification of the stochastic volatility models that follow. Specifically, the null hypotheses are all one-sided and test whether (for example) the returns of January are equal versus higher (or for other calendar effects lower) than the rest of the months. Each hypothesis has been tested at $90 \%, 95 \%$, and $99 \%$ confidence levels. The details on forming one-sided hypothesis tests using bootstrapping are in Appendix A (also see Hansen (2003)).

Tables $2 \mathrm{~A}$ and $2 \mathrm{~B}$ demonstrate that the null hypothesis is only rejected for the Tuesday and Wednesday returns with $90 \%$ confidence, and for the September returns with $95 \%$ confidence. This is a striking new result based on the bootstrapping of the seasonal sample means. Among the 18 seasonal dummies examined, only three are statistically significant in their average returns. In fact, two of the three (Tuesday and Wednesday) are significant only at the low level of $\alpha=10 \%$, and then become insignificant when excluding the three Crash of 1987 outliers (see Table 2C). None of the calendar effects discussed extensively in the asset pricing literature such as Monday, Holiday, January and October are actually statistically significant in the daily returns of the S\&P 500 series!

These results change drastically when we test for seasonal heteroscedasticity. For the day of the week effect in absolute daily returns (a model-free proxy of volatility) Monday is rejected with $95 \%$ confidence, whereas Wednesday and $H O L$ with $99 \%$ confidence. For the month of the year effect in absolute daily returns, May, June, July and October are rejected with $99 \%$ confidence and December with $90 \%$ confidence. Hence many of the seasonal effects that the asset pricing literature has investigated in expected returns turn out to be statistically significant only in volatility, namely Monday, Wednesday, Holiday and October. It is important to note that as Tables $2 \mathrm{C}$ and $2 \mathrm{D}$ demonstrate, these results are not driven by the Crash of 1987 and tend to remain valid for four different subsamples examined. In particular, removing the three most volatile days of the sample (October 19-21) does not change the results noticeably: the volatilities of Wednesday and October remain statistically different to their complements with $99 \%$ confidence, and the null for Monday's volatility is now rejected with $90 \%$ confidence instead of $95 \%$. Further, all seven calendar effects which are statistically 
significant for the full sample remain significant for at least two of the four subsamples, while three seasonals are so for three of the four subsamples. Interestingly, January is the one prominent calendar effect, which is statistically significant for neither returns nor volatility and this is true for all four subsamples.

Figure 1 displays the seasonal heteroscedasticity of the S\&P 500 index by graphing the $\mathrm{u}$-shaped day of the week and the month of the year average absolute returns over the entire sample. Figures 2 and 3 isolate the effects of Monday and Wednesday on the one hand, and January, October and $H O L$ on the other. These two figures offer a visual inspection of (i) the overlap of the two bootstrap distributions for each seasonal mean and its complement, and (ii) the position of a sample mean in the bootstrap distribution of its complement. For Monday, Wednesday and October we also graph the bootstrap distributions excluding the three most volatile days of the sample (October 19-21) and thus provide a visual illustration of the fact that the statistical significance of these three seasonal volatility effects is robust to the three day exclusion. In short, there is strong quantitative and visual evidence that for the dataset examined here, there is clear misspecification if we assume that the volatility of all days and months persists around the same constant. It is important to note that we do not argue that the seasonal effects remain constant over time or that the stronger effects are statistically significant every single time period. On average, however, seasonality in volatility is strong and statistically significant throughout the entire sample.

\section{Stochastic Volatility}

\subsection{The Plain Vanilla SV model}

In the stochastic volatility (SV) framework, the plain vanilla SV model presents the benchmark against which model comparisons will be conducted. According to this plain vanilla SV benchmark, the daily S\&P 500 returns are assumed to follow a univariate discrete-time AR(1) process and are driven by Gaussian innovations:

$$
\begin{gathered}
y_{t}=\alpha+\rho y_{t-1}+\omega_{t} \\
\omega_{t}=\varepsilon_{t} v_{t}, \quad \varepsilon_{t} \sim \operatorname{NID}(0,1)
\end{gathered}
$$

The persistence of the stochastic conditional volatility $v_{t}$ is captured by the latent $\log$ variance process $h_{t}$, which is modelled as a dynamic Gaussian variable:

$$
\begin{gathered}
v_{t}=\exp \left(h_{t} / 2\right) \\
h_{t}=\mu+\beta^{\prime} X_{t}+\phi\left(h_{t-1}-\mu\right)+\sigma \eta_{t}, \quad \eta_{t} \sim N I D(0,1)
\end{gathered}
$$


In the SV model, return and volatility innovations are independent: $\left\{\varepsilon_{t}\right\} \perp\left\{\eta_{t}\right\}$. Further, the model assumes (and the estimation algorithm imposes) $|\rho|,|\phi|<1$ so that both returns and their volatility are stationary processes. ${ }^{7}$ Finally, all SV specifications examined in this paper reduce $\beta^{\prime} X_{t}=\beta_{1} y_{t-1}$. In words, if $\beta_{1}<0$ the lower the return shock, the higher the conditional variance in the next few periods. This simple specification allows for a level component measured by $\beta_{1} y_{t-1}$, as well as a dynamic component measured by $\phi^{j} \beta_{1} y_{t-j}$. In the following sections, the plain vanilla SV specification is generalized to account for the fat tails (excess kurtosis), and seasonal periodicity of the daily S\&P 500 returns and volatility in the form of day of the week, holiday, and month of the year periodic calendar effects.

\subsection{SV with Fat Tails $(S V t)$}

Excess kurtosis is a highly documented higher order moment property of daily equity returns, which deviates from Gaussian behaviour. The presence of fat tails in daily returns implies a positive probability that very large positive or negative shocks may occur. For example, in the case of the S\&P 500 daily returns sample examined here, such shocks are up to 23 standard deviations below, and up to 8 standard deviations above the daily return mean. A standard way of modelling fat tails is by assuming that the daily innovations follow a Student- $t$ distribution:

$$
\omega_{t}=u_{t} v_{t}, \quad u_{t} \sim \operatorname{iid} t(0,1, \nu)
$$

This assumption defines the $S V t$ model.

The univariate SV models with either Gaussian or Student-t innovations can be put in a convenient state-space form. Specifically, Kim, Shephard and Chib (1998) take a logarithmic transformation of the observed returns data and approximate the $\log \chi^{2}(1)$ distribution by a seven-component mixture of normal densities. Then, both the transformed data and the log-variance equations are linear and conditionally Gaussian. This transformation to a conditionally Gaussian state-space model via a mixture of normals approximation allows the use of the Kalman filter and has been fundamentally important in the development of efficient MCMC estimation procedures (see Appendix B for some of the details).

\subsection{Periodic Stochastic Volatility (PSV)}

This paper introduces a set of periodic SV specifications in which the constants (levels) in both the conditional mean and the conditional variance are generalized to account for three distinct types of seasonality: day of the week, holiday (non-trading day) and month of the year effects. In the PSV framework, we explore the three following specifications with both Gaussian and Student- $t$ innovations.

\footnotetext{
${ }^{7}$ In practice, the stationarity restrictions are never violated for daily returns data.
} 


\subsubsection{A Comprehensive Specification with a Cyclical Month of the Year Effect $\left(P S V_{C}\right)$}

In the high-dimensional specification we denote as $P S V_{C}$, the mean has a simple day of the week and holiday effect:

$$
\begin{gathered}
\alpha=\alpha_{t}=\alpha_{D, t}+\alpha_{M, t} \\
\alpha_{D, t}=\delta_{0}+\delta_{1} M O N_{t}+\delta_{2} T U E_{t}+\delta_{3} W E D_{t}+\delta_{4} T H U_{t}+\delta_{5} H O L_{t}
\end{gathered}
$$

and a parsimonious cyclical six-term month of the year effect ${ }^{8}$

$$
\begin{aligned}
\alpha_{M, t}= & \delta_{6} \sin \left(\frac{2 \pi}{12} m_{t}\right)+\delta_{7} \cos \left(\frac{2 \pi}{12} m_{t}\right)+\delta_{8} \sin \left(\frac{4 \pi}{12} m_{t}\right) \\
& +\delta_{9} \cos \left(\frac{4 \pi}{12} m_{t}\right)+\delta_{10} \sin \left(\frac{6 \pi}{12} m_{t}\right)+\delta_{11} \cos \left(\frac{6 \pi}{12} m_{t}\right)
\end{aligned}
$$

where $H O L_{t}$ is a dummy variable for the day which follows a non-trading day, that is a weekday which follows a weekday for which the market was closed (i.e. $H O L_{t}$ is a postholiday). All the day dummies are in $\{0,1\}$, and $m_{t} \in\{1,2, \ldots, 12\}$. Note that the constant of the Fourier expansion is absorbed by $\delta_{0}$.

Similarly, the volatility process is generalized to account for the three seasonal effects:

$$
\begin{gathered}
v_{t}=l_{t} \exp \left(h_{t} / 2\right), \quad l_{t}=\exp \left(\gamma_{t} / 2\right) \\
\gamma_{t}=\gamma_{D, t}+\gamma_{M, t} \\
\gamma_{D, t}=\gamma_{1} M O N_{t}+\gamma_{2} T U E_{t}+\gamma_{3} W E D_{t}+\gamma_{4} T H U_{t}+\gamma_{5} H O L_{t} \\
\gamma_{M, t}=\gamma_{6} \sin \left(\frac{2 \pi}{12} m_{t}\right)+\gamma_{7} \cos \left(\frac{2 \pi}{12} m_{t}\right)+\gamma_{8} \sin \left(\frac{4 \pi}{12} m_{t}\right) \\
+\gamma_{9} \cos \left(\frac{4 \pi}{12} m_{t}\right)+\gamma_{10} \sin \left(\frac{6 \pi}{12} m_{t}\right)+\gamma_{11} \cos \left(\frac{6 \pi}{12} m_{t}\right)
\end{gathered}
$$

${ }^{8}$ Any periodic function $f\left(m_{t}\right)$ can be represented by $f\left(m_{t}\right)=\sum_{j=0}^{6}\left[a_{j} \sin \left(\frac{2 \pi j}{12} m_{t}\right)+b_{j} \cos \left(\frac{2 \pi j}{12} m_{t}\right)\right]$, where $a_{j}$ and $b_{j}$ are the Fourier coefficients. These are the regression coefficients obtained by regressing $f\left(m_{t}\right)$ on $\sin \left(\frac{2 \pi j}{12} m_{t}\right)$ and $\cos \left(\frac{2 \pi j}{12} m_{t}\right)$. In notation local to this footnote, we use the approximation $f\left(m_{t}\right)=b_{0}+a_{1} \sin \left(\frac{2 \pi}{12} m_{t}\right)+b_{1} \cos \left(\frac{2 \pi}{12} m_{t}\right)+a_{2} \sin \left(\frac{4 \pi}{12} m_{t}\right)+b_{2} \cos \left(\frac{4 \pi}{12} m_{t}\right)+a_{3} \sin \left(\frac{6 \pi}{12} m_{t}\right)+b_{3} \cos \left(\frac{6 \pi}{12} m_{t}\right)$, where $b_{0}$ is absorbed by the constant of the return equation. Clearly, the three frequencies used are $\omega_{j}=\frac{2 \pi j}{12}$ for $j=\{1,2,3\}$. The phase of this Fourier process is equal to $b_{0}+b_{1}+b_{2}+b_{3}$, which denotes where the Fourier cycle is at $m_{t}=0$. For more details on the Flexible Fourier Form (FFF) see Gallant (1981). 
Note that there is no need for a constant in $\gamma_{M, t}$ because it is absorbed by $\mu$, which is the "fundamental" or acyclical component of the log-variance $h_{t}$. In words, the conditional standard deviation of the $P S V_{C}$ specification is subject to the seasonal level effect $l_{t}$, which is separate from the dynamic component $\exp \left(h_{t} / 2\right)$.

Clearly, the $P S V_{C}$ model is designed to capture all three calendar effects in both the first and the second conditional moments using (in the context of the SV framework) the lowest parameter dimension possible. The purpose of the $P S V_{C}$ specification is not only to isolate the exact effect of each day and month, but more importantly to test whether adjusting the return and volatility levels to fully account for seasonal periodicity results in superior performance.

The key to this specification is the six-term Fourier approximation of the month of the year effect. The dimension of this approximation was chosen such that it fits the monthly seasonality reasonably well, while using substantially less parameters than a series of eleven monthly dummies. The six-term Fourier approximation is shown in Figure 4. For the $P S V_{C}$ specification, the MCMC algorithm must provide estimates of the four sets of parameters $\theta=\left\{\theta_{1}, \nu, \theta_{2}, \theta_{3}\right\}$. Here, $\theta_{1}=\left\{\delta_{0}, \rho\right\}$ is the set of parameters of the conditional mean, $\theta_{2}=\left\{\mu, \beta_{1}, \phi, \sigma^{2}\right\}$ is the set of parameters of the Gaussian log-variance process, and $\theta_{3}=$ $\left\{\delta_{j}, \gamma_{j}\right\}, 1 \leq j \leq 11$ are the dummy and Fourier coefficients in the mean and the conditional $\log$-variance, respectively. All $\theta$ parameters are time invariant.

\subsubsection{Two Parsimonious Periodic Specifications $\left(P S V_{L}, P S V_{H}\right)$}

It is useful to also examine two parsimonious low-dimensional $P S V$ specifications, which selectively account for the stronger seasonal return and volatility effects. In the case of the $P S V_{L}$ model, the mean has the following specification:

$$
\alpha=\alpha_{t}=\delta_{0}+\delta_{1} M O N_{t}+\delta_{2} W E D_{t}+\delta_{3} H O L_{t}+\delta_{4} J A N_{t}+\delta_{5} O C T_{t}
$$

Clearly, $\delta_{0}$ is the constant for any day other than Monday, Wednesday and $H O L$, and any month other than January and October.

Similarly, the volatility seasonal level effect of $P S V_{L}$ is:

$$
\gamma_{t}=\gamma_{1} M O N_{t}+\gamma_{2} W E D_{t}+\gamma_{3} H O L_{t}+\gamma_{4} J A N_{t}+\gamma_{5} O C T_{t}
$$

Isolating the Monday, Wednesday, and Holiday effects on the one hand, and the January and October effects on other hand, clearly provides for a parsimonious $P S V$ model. Note that the January and October volatility scale factors are consistent with the specification of Glosten, Jagannathan and Runkle (1993). More importantly, this parsimonious specification allows for testing how well the $P S V_{L}$ model performs against both the cyclical $P S V_{C}$ specification and the aperiodic plain vanilla SV model.

We define $P S V_{H}$ as the second parsimonious specification, which only conditions on the strongest and highly statistically significant seasonal effect, the non-trading day $H O L$ effect. 
The $P S V_{H}$ model ignores day of the week and month of the year effects and is therefore highly parsimonious. The mean and log-variance levels are simply

$$
\begin{gathered}
\alpha=\alpha_{t}=\delta_{0}+\delta_{1} H O L_{t} \\
\gamma_{t}=\gamma_{1} H O L_{t}
\end{gathered}
$$

\subsubsection{Seasonality and Fat Tails $(P S V t)$}

All three $P S V$ specifications presented above assume Gaussian return innovations, but have an equivalent fat-tailed representation which assumes Student- $t$ innovations. Hence we specify $P S V t_{C}, P S V t_{L}$, and $P S V t_{H}$. The fat-tailed periodic specifications are instrumental in examining the relation between fat tails and seasonality.

\section{Testing Hypothesis}

In Section 2, we conducted model-free testing of the statistical significance of seasonal periodicity and seasonal heteroscedasticity using bootstrapping. In this section, we discuss a set of testing hypotheses, which are based on appropriately specified stochastic volatility models. We will explore two types of tests: (i) measuring whether the set of estimated Student- $t$ and periodic parameters are statistically different from zero, and (ii) assessing the overall performance of all SV specifications by ranking them using the toolkit discussed in the next two sections.

\subsection{Parameter Significance}

The Gaussian and fat-tailed stochastic volatility specifications discussed thus far $\left\{S V, P S V_{C}\right.$, $\left.P S V_{L}, P S V_{H}\right\}$ and $\left\{S V t, P S V t_{C}, P S V t_{L}, P S V t_{H}\right\}$, respectively, provide a framework for formally testing three hypotheses on parameter significance. First, does the conditional Student- $t$ distribution fit the data better than a Gaussian? In other words, do we get low values for the estimate of the degrees of freedom parameter $\nu$ ? Second, does a full account of the periodic seasonal level effects in both the first and the second time-varying moments result in large seasonal coefficients, which are statistically different from zero? Further, are there parsimony gains from reducing the high dimension of $P S V_{C}$ to the lower dimension of $P S V_{L}$, and even more so $P S V_{H}$ ? Third, is there interaction between fat tails and seasonal heteroscedasticity? Specifically, does the conditioning on day of the week, holiday, and month of the year effects reduce or eliminate the need for fat-tailed SV specifications? These are summarized by three separate null hypotheses:

$$
H_{0}: \quad \text { Plain-Vanilla } S V
$$

or 


$$
\begin{array}{clll}
H_{0}^{A}: & \nu>30 ; & \text { No Fat Tails } \\
H_{0}^{B}: & \delta_{j}=\gamma_{j}=0 \forall j ; & \text { No Seasonality } \\
H_{0}^{C}: & H_{0}^{A} \text { and } H_{0}^{B} & \\
& \\
& H_{1}: \quad \text { otherwise }
\end{array}
$$

\subsection{Performance Evaluation}

We assess the conditional dynamics of all SV specifications by computing one-step ahead density forecasts. We evaluate the relative performance of each specification by ranking all SV models according to the likelihood-based Bayes factor criterion. Specifically, we examine four questions, which we answer by comparing the performance of the following four sets of models:

- Should we condition on the three seasonal effects? ( $S V$ vs. three $P S V$ specifications)

- Should we use the Student-t distribution? ( $S V$ vs. $S V t$ )

- What is the relative importance of fat tails and seasonality? ( $S V t$ vs. three $P S V$ specifications)

- Are fat tails necessary after conditioning on seasonality? (three $P S V$ vs. three $P S V t$ specifications)

For all four questions, we will examine both the full sample and an adjusted sample which excludes the three extreme outliers of October 19-21, 1987. These three dates capture the Crash effect, as October 19 is the minimum return and October 21 is the maximum return in the sample. This is of interest as we need to determine the extent to which the fat tails on the one hand, and the Monday, Tuesday, Wednesday and October effects on the other hand, are driven by the three observations. The set of tools we use in order to answer these questions are presented in the next two sections.

\section{Bayesian MCMC Estimation}

We perform Bayesian MCMC estimation by constructing a Markov chain whose limiting distribution is the target posterior density of interest. This Markov chain is a Gibbs sampler in which all parameters are drawn sequentially from their full conditional posterior distribution. The chain is then iterated a large number of times and the sampled draws, beyond a burn-in period, are treated as variates from the target distribution. 
For example, in the case of the high-dimensional $P S V t_{C}$ model, the MCMC algorithm produces estimates of the posterior means of (i) the parameters of the return equation $\left\{\theta_{1}, \nu\right\}$, where $\theta_{1}=\{\alpha, \rho\}$, (ii) the log-volatility parameters $\theta_{2}=\left\{\mu, \beta_{1}, \phi, \sigma^{2}\right\}$, and (iii) the seasonal parameters $\theta_{3}=\{\gamma, \delta\}$ for the day of the week, holiday, and month of the year effects in both the mean $\left\{\delta_{j}\right\}$ and the variance $\left\{\gamma_{j}\right\}, j \leq 11$.

The key to estimating the $P S V$ models is the efficient sampling of the seasonal level effects in the conditional variance. The paper adds a simple Gibbs step to the Chib, Nardari and Shephard (2002) algorithm, in which the $\gamma=\left\{\gamma_{j}\right\}$ vector is drawn conditional on the logvolatilities $\left\{h_{t}\right\}$ using a precision-weighted average of prior information and the conditional likelihood. Despite its simplicity, this method is numerically superior to the sampling of $\gamma$ in the same block as the log-volatility parameters $\theta_{2}$ because it avoids the numerical problems which may arise from high-dimensional optimization. The results indicate that the simple Gibbs step is highly efficient. The details on sampling $\left\{\gamma_{j}\right\}$ are summarized in Appendix B.

\subsection{MCMC Diagnostics}

The mean of the MCMC draws is an asymptotically efficient estimator of the posterior mean of $\theta$ (see Geweke (1989)). The Numerical Standard Error (NSE) is the square root of the asymptotic variance of the estimator:

$$
\begin{gathered}
N S E=\sqrt{\frac{\widehat{S}}{M}} \\
\widehat{S}=\widehat{\gamma}_{0}+2 \sum_{j=1}^{B_{M}} K(z) \widehat{\gamma}_{j}
\end{gathered}
$$

Here, $M=5,000$ is the number of iterations (beyond the initial burn-in of 1,000 iterations), $j=1, \ldots, B_{M}=500$ lags is the set bandwidth, $z=\frac{j}{B_{M}}$, and $\widehat{\gamma}_{j}$ is the sample autocovariance of the MCMC draws of each estimated parameter cut according to the Parzen kernel $K(z)$.

The NSE diagnostic is distinct from the MCMC standard deviation reported in Tables 3 through 7 . The latter is simply a measure of the variation in the MCMC parameter draws. In contrast, NSE is a measure of variation of the posterior mean estimate across many MCMC chains that can be potentially run. In other words, NSE measures how much difference one should expect in the estimate of the posterior mean if the experiment were to be repeated, and hence provides a measure of convergence.

The Relative Numerical Inefficiency (RNI) is given by

$$
R N I=1+2 \sum_{j=1}^{B_{M}} K(z) \rho(j)
$$

where $\rho(j)$ is the autocorrelation in the MCMC draws at lag $j$ for the parameter of interest. RNI accounts solely for the variance inflation (inefficiency) due to the serial correlation of 
the MCMC parameter draws (see Geweke (1992) for the details). In general, the lower the serial correlation, the lower the number of iterations needed to attain a given level of numerical accuracy. For example, if RNI were to be halved, one would need half the number of iterations to attain the same level of numerical accuracy. The relatively low RNI values reported in Tables 3-7 reflect the efficiency of the two separate Metropolis-Hastings algorithms used to sample $\theta_{2}$ and $\nu$.

\subsection{Volatility Estimates}

The conditional dynamics of the SV model are essentially driven by the persistent, latent and Gaussian log-volatility process $\left\{h_{t}\right\}$. The tools of Chib, Nardari and Shephard (2002) allow the simulation of three distinct estimates of the $\left\{h_{t}\right\}$ vector. First is the smoothed volatility. The MCMC chain samples from the density $h^{(i)} \mid F_{T}, \theta^{(i)}$. In words, it samples the $\left\{h_{t}^{(i)}\right\}$ vector at a given iteration $i=1, \ldots, M$ conditional on the information $F_{T}$ from the full dataset (hence smoothed) and the parameter vector draw $\theta^{(i)}$.

Second is the filtered volatility. The Auxiliary Particle Filter of Pitt and Shephard (1999) samples from the density $h_{t}^{j} \mid F_{t}, \theta$. In words, it generates a $j=1, \ldots, M=2,000$ vector of $\log$-volatilities (the "particles") at each $t$, given the information set $F_{t}$ and the true values of $\theta$ proxied by the MCMC posterior mean estimates. This is a non-trivial task performed by an Auxiliary Sampling-Importance Resampling algorithm. The SV application of the algorithm is also detailed in Chib, Nardari and Shephard (2002).

Third is the one-step ahead predictive volatility. This samples from $h_{t+1}^{j} \mid F_{t}, \theta$. Given a vector of $j=1, \ldots, M=2,000$ particles from the filtered density $h_{t}^{j} \mid F_{t}, \theta$ it is straightforward to compute the one-step ahead vector of particles from the predictive density using the Gaussian evolution equation:

$$
h_{t+1}^{j}=\mu+\beta^{\prime} X_{t}+\phi\left(h_{t}^{j}-\mu\right)+\sigma \eta_{t+1}^{j}, \quad \eta_{t+1}^{j} \sim N I D(0,1)
$$

\subsection{Log-Likelihood}

The likelihood function of SV models is not available analytically and hence must be simulated.

$$
L(\theta ; y)=f\left(y_{1, \cdots,} y_{T} \mid F_{0}, \theta\right)=\prod_{t=1}^{T} f\left(y_{t} \mid F_{t-1}, \theta\right)
$$

Specifically, the log-likelihood function is evaluated under the predictive density as

$$
\log \widehat{L}=\sum_{t=1}^{T} \log \widehat{f}\left(y_{t} \mid F_{t-1}, \theta\right)=\sum_{t=1}^{T} \log \widehat{f}_{t}\left(y_{t} \mid h_{t}, \theta\right)
$$

where $h_{t}$ is the one-step ahead predictive volatility $h_{t} \mid F_{t-1}, \theta$, and $\theta$ is taken as the posterior mean estimate from the MCMC simulations. 


\section{Performance Evaluation}

A statistical model will not be empirically successful unless it is well specified. For example, a risk manager may be interested in the average probability with which an event arises. This is determined by a well-specified unconditional distribution. More importantly, however, managing day-to-day risk involves making decisions conditional on all available information at time $t$. This requires a well-specified conditional distribution. This section formally tests whether the $P S V$ models (i) perform better in capturing the in-sample and out-of-sample conditional dynamics of the daily S\&P 500 returns and volatility period-by-period, and (ii) are better specified than the $S V$ and $S V t$ benchmarks using Bayes factors as the criterion for model selection.

\subsection{Density Forecasts and Conditional SV Dynamics}

Kim, Shephard and Chib (1998) form a set of diagnostic tests for assessing the adequacy of the conditional distribution of SV models using the simple Rosenblatt (1952) transformation. This defines SV residuals as

$$
u_{t+1}=\operatorname{Pr}\left(Y_{t+1} \leq y_{t+1} \mid h_{t+1}, \theta\right) \sim U I D[0,1]
$$

where $y_{t+1}$ is the ex-post realized return and $\theta$ is the posterior mean estimate. The probability is evaluated using the ex-ante forecasted cumulative distribution function, where $\left\{h_{t+1}\right\}$ is the one-step ahead predictive log-volatility $h_{t+1} \mid F_{t}, \theta$. The uniform residuals are then mapped to a normal distribution simply because there is a larger battery of specification tests available for a normal random variable. Then, under the null that the model is correctly specified, $n_{t+1}=f^{-1}\left(u_{t+1}\right)$ should be Gaussian white noise. Note that the normalized residuals $\left\{n_{t+1}\right\}$ contain the same information as the uniform $\left\{u_{t+1}\right\}$.

Using these normalized residuals as a basis for diagnostic testing is not restricted to $\mathrm{SV}$ models. Berkowitz (2001) suggests the use of $n_{t+1}$ (also known as density forecasts) for evaluating the performance of generic risk models. An important advantage of density forecasts is that there are as many of them as data observations. In contrast, for example, the popular value-at-risk $(\mathrm{VaR})$ calculation measures the frequency of tail events and hence produces too few tail observations for reasonable sample sizes. Further, note that density forecasts also account for the size of observations, not just their frequency. In short, therefore, density forecasts offer statistical power that is missing in VaR calculations, while using information from the entire conditional distribution, not just a single quantile.

Tables 8A, 8B, and 8C report the specification tests for the in-sample and out-of-sample $\left\{n_{t+1}\right\}$ diagnostics. We follow the notation of Bowman and Shenton (1975) and define $\sqrt{b_{1}}=$ $m_{3} / m_{2}^{3 / 2}$ and $b_{2}=m_{4} / m_{2}^{2}$, where $m_{j}$ is the $j t h$ centralized sample residual moment. Then, we define $S K E W$ and $K U R T$ to be the asymptotic standard normal test statistics of $\sqrt{b_{1}}$ and $b_{2}$ respectively: 


$$
\begin{gathered}
S K E W=\sqrt{\frac{T}{6}} \sqrt{b_{1}} \sim N(0,1) \\
K U R T=\sqrt{\frac{T}{24}}\left(b_{2}-3\right) \sim N(0,1)
\end{gathered}
$$

The identification of skewness and excess kurtosis in the density forecasts is very important. As it becomes increasingly difficult to capture the properties of higher order moments, misspecification occurs more often at the tails of the predictive density. It is straightforward to prove that if the observed data $y_{t}$ is fat-tailed relative to the SV model, then the density forecasts will be fat-tailed relative to the standard normal. ${ }^{9}$

\subsection{Model Risk and Bayes Factors}

Model risk arises from the uncertainty over selecting a model specification. Bayes factors can account for model risk by providing a framework for specification diagnostics over a set of given models. Specifically, a Bayes factor offers a summary of the evidence provided by the data in favour of a scientific theory represented by a statistical model. ${ }^{10}$ Consider the two competing hypotheses (models) $M_{1}$ and $M_{2}$. Using Bayes theorem, it is straightforward to show that the Bayes factor $B_{21}$ (in favour of model $M_{2}$ ) is the ratio of posterior to prior odds

$$
B_{21}=\frac{p\left(M_{2} \mid y\right)}{p\left(M_{1} \mid y\right)} \frac{\pi\left(M_{1}\right)}{\pi\left(M_{2}\right)}
$$

and is computed as the ratio of the marginal likelihoods

$$
B_{21}=\frac{p\left(y \mid M_{2}\right)}{p\left(y \mid M_{1}\right)}
$$

The marginal likelihood of a model is defined as

$$
p\left(y \mid M_{j}\right)=\int_{\theta} p\left(y, \theta \mid M_{j}\right) d \theta=\int_{\theta} p\left(y \mid \theta, M_{j}\right) \pi\left(\theta \mid M_{j}\right) d \theta
$$

Note that the marginal likelihood is an averaged and not a maximized likelihood. This implies that the Bayes factor is an automatic "Occam's Razor" in that it integrates out parameter uncertainty. ${ }^{11}$ Further, the marginal likelihood is simply the normalizing constant of the posterior density. Suppressing the model index $M_{j}$ for simplicity, the marginal likelihood can be written as

\footnotetext{
${ }^{9}$ For the proof see Berkowitz (2001).

${ }^{10}$ See Kass and Raftery (1995) for a review of Bayes factors.

${ }^{11}$ Occam's razor is just the principle of parsimony. For an econometrician, the most useful statement of the principle is "among two competing theories, which make exactly the same prediction, the simplest one is best".
} 


$$
p(y)=\frac{f(y \mid \theta) \pi(\theta)}{\pi(\theta \mid y)}
$$

where $f(y \mid \theta)$ is the likelihood, $\pi(\theta)$ the prior density, $\pi(\theta \mid y)$ the posterior density, and $\theta$ is evaluated at the posterior mean estimate $\theta^{*}$. Since $\theta$ is drawn in the context of Gibbs MCMC sampling, the posterior density $\pi(\theta \mid y)$ is computed using the technique of reduced conditional MCMC runs of Chib (1995). For the parameter blocks of $\theta$ (the log-variance parameters and the degrees of freedom) which are sampled in the MCMC chain by implementing a MetropolisHastings algorithm, the posterior density is computed as in Chib and Jeliazkov (2001).

To assess the information provided by a Bayes factor, it is useful to consider twice its natural logarithm so as to be on the same scale as the likelihood ratio statistics. To make the interpretation more familiar, Table 10A presents the range of the values of $2 \ln \left(B_{21}\right)$ that constitute evidence against the null hypothesis $M_{1}$. Finally, note that model comparisons based on Bayes factors are asymptotically equivalent to evaluations based on the Schwartz (or equivalently the BIC) criterion. $^{12}$

\section{Results and Discussion}

\subsection{Parameter Estimates}

Tables 3 through 7 present the MCMC posterior mean estimates for the parameters of all $S V$, $S V t, P S V$ and $P S V t$ specifications examined in this paper. As seen in Table 3, there are four benchmark properties for the plain vanilla SV parameter estimates when applied to the daily S\&P 500 returns: (i) low persistence in the mean $(\rho=0.052)$, (ii) very high persistence in the conditional $\log$-variance $(\phi=0.969)$, (iii) a negative relationship between volatility and lagged returns $\left(\beta_{1}=-0.088\right)$, and (iv) an important stochastic component in the conditional $\log$-variance $\left(\sigma^{2}=0.028\right.$ or $\left.\sigma=0.167\right)$. All parameters are well estimated as indicated by their low RNI and close to zero NSE values. In general, for all aperiodic and periodic specifications we run, the non-seasonal parameter estimates for persistence, leverage and stochastic variance are very similar to the benchmark SV case. Hence we will now focus exclusively on the added value of each $P S V, S V t$ and $P S V t$ specification relative to the plain vanilla SV. In particular, we will emphasize the three following strong results.

In the $S V t$ model, the role of the degrees of freedom parameter $\nu$ is to capture the large excess kurtosis due to the frequent outliers in the unconditional distribution of daily returns. The MCMC posterior mean estimates for $\nu$ are presented in Tables $4 \mathrm{~A}$ and $4 \mathrm{~B}$. As expected, the posterior mean of $\nu$ is relatively low $(\nu \approx 11)$ for the full sample. Surprisingly, $\nu$ remains

\footnotetext{
${ }^{12}$ The Schwartz criterion is defined as $S=\log p\left(y \mid \widehat{\theta}_{2}, M_{2}\right)-\log p\left(y \mid \widehat{\theta}_{1}, M_{1}\right)-\frac{1}{2}\left(d_{2}-d_{1}\right) \log (T)$, where $d_{j}$ is the dimension of $\theta_{j}$. As $T \rightarrow \infty$ the Schwartz criterion satisfies $\frac{S-\log B_{21}}{\log B_{21}} \rightarrow 0$ and thus may be viewed as a rough approximation to the $\log$ of the Bayes factor. Note that $B I C=-2 S$. Again, see Kass and Raftery (1995) for the details.
} 
low $(\nu \approx 12)$ even when we re-estimate the $S V t$ model on the same data but exclude the three days of the Crash of 1987 (October 19-21). Therefore, the $\nu$ estimate is robust to exclusion of the largest outliers in the data.

Without exception, for all PSVt specifications, the inclusion of the seasonal level effects in volatility essentially eliminates the need for a Student- $t$ distribution. Even in the presence of only the (very statistically significant) holiday effect, the degrees of freedom parameter jumps from a value of $\nu \approx 11$ in $S V t$ to a Gaussian-like value ${ }^{13}$ of $\nu \approx 38$ in $P S V t_{H}$. The addition of more seasonals reduces even further the need for fat tails: the more seasonals we accounted for, the higher the value of $\nu\left(\nu \approx 47\right.$ for $P S V t_{L}$ and $\nu \approx 52$ for $\left.P S V t_{C}\right)$. These high $\nu$ estimates appear to be statistically significant ${ }^{14}$ since in all cases the $5 \%$ lower percentile of the MCMC draws for the degrees of freedom is very close to a Gaussian value of 30. Further, consistent with one of the main themes in this paper, these results are robust to the exclusion of the three Crash of 1987 days. The exclusion raises the $\nu$ values slightly, but all conclusions relevant for the full sample are still valid. In conclusion, the combination of the low $\nu$ estimate for $S V t$, its lack of sensitivity to the 1987 outliers, and especially the massive effect of seasonal heteroscedasticity on fat tails provide the first strong result of the evidence offered by the parameter estimates.

Having established the effect of seasonal heteroscedasticity on fat tails, Tables 5, 6 and 7 present the MCMC posterior means for the seasonal parameters in the mean and log-variance of the Gaussian $P S V_{C}, P S V_{L}$, and $P S V_{H}$ specifications. Perhaps the easiest specification to interpret is the parsimonious $P S V_{L}$ model, which conditions both returns and their logvariance on Monday, Wednesday, Holiday, January and October effects. In this model, January has the highest positive effect on the conditional mean $\left(\delta_{4}=0.053\right)$, and $H O L$ the highest negative effect $\left(\delta_{3}=-0.081\right)$. In fact, a common theme across all three seasonal models is that the holiday effect is by far the strongest in both returns and volatility. In the $P S V_{L}$ model, $H O L$ raises the level of the conditional variance by a factor of $\exp \left(\gamma_{3}\right)=1.54$. This is much higher than the Monday volatility effect $\left(\exp \left(\gamma_{1}\right)=1.09\right)$, the January volatility effect $\left(\exp \left(\gamma_{3}\right)=1.24\right)$, and even the October volatility effect $\left(\exp \left(\gamma_{4}\right)=1.25\right)$. As expected, Wednesday lowers the conditional variance by a factor of $\exp \left(\gamma_{2}\right)=0.88$. The strength of the periodic volatility effects provides the second major finding offered by the evidence. Note that in the full parametric $P S V_{L}$ model, the January volatility effect is strong and statistically significant even though the bootstrapping evidence did not provide such strong support.

Similar to our model-free discussion of the seasonal properties of the data using bootstrap-

\footnotetext{
${ }^{13}$ For values $\nu>30$ the Student- $t$ distribution becomes indistinguishable from the Gaussian. From a technical point of view, note that the higher the value for $\nu$, the more the Student- $t$ distribution resembles the Gaussian and hence the more difficult it is to estimate the exact value for $\nu$. This is reflected in the large NSE and RNI values for the $\nu$ estimates of the PSVt models.

${ }^{14}$ Ideally, in Bayesian estimation we measure the statistical significance of the estimated parameters by forming a Bayesian confidence interval. However, this requires full specification of the marginal posterior distribution, which for the SV parameters is not available analytically. Indeed, this is the reason why we perform MCMC estimation in the first place.
} 
ping, perhaps the most important aspect of the parameter estimates is not their size but their statistical significance. In the Bayesian MCMC framework, we assess statistical significance by reporting the $5 \%$ and $95 \%$ percentiles of the MCMC draws and examine whether the range of $90 \%$ of the draws includes zero ${ }^{15}$. The third and final finding on the parameter estimates is consistent with the bootstrapping results: none of the seasonal coefficients in the mean (even in the comprehensive $P S V_{C}$ model) are statistically different from zero! In contrast, all the seasonal parameters in the log-variance of the $P S V_{L}$ model are quite statistically different from zero (with the exception of Monday, which is at the very margin). Interestingly, for the $P S V_{C}$ specification only one of the six Fourier coefficients is statistically different from zero leading us to believe that modelling the seasonal behaviour of all 12 months is not necessary as not all are statistically significant in the data.

\subsection{Bayes Factor Diagnostics and Density Forecasts}

All SV specifications are ranked by the likelihood-based Bayes factor criterion. The Bayes factor calculations are shown in Table 10B and present us with a very strong result. Without exception, the Bayes factor rankings are purely driven by the degrees of freedom estimates: the lower the estimated $\nu$, the higher the ranking of the model. In other words, $S V t$ is ranked first and SV last. Following $S V t$ are all the $P S V t$ models, where $P S V t_{H}(\nu=38)$ is second, $\operatorname{PSVt}_{L}(\nu=47)$ is third, and $P S V t_{C}(\nu=52)$ is fourth. This result is in stark contrast to the idea behind the high estimated values for the degrees of freedom parameter $\nu$ of the $P S V t$ specifications. For the 22 year sample of daily S\&P 500 data, the PSVt estimates of $\nu$ are consistent with the Gaussian distribution. Therefore, whereas the data indicates that a Student- $t$ specification is not necessary when we explicitly model seasonal heteroscedasticity, the likelihood (and hence the Bayes factors) favours the model with the lowest $\nu$. We believe that this is a general weakness of likelihood-based measures, which just tend to magnify the effect of outliers, and it does not invalidate the effect of seasonal heteroscedasticity on fat tails. For example, we recomputed a set of artificial values for the PSVt log-likelihood using the estimated $P S V t$ parameters and an artificially set $\nu=11.11$ (because it is equal to that of $S V t$ ). Now, the PSVt models dominate the $S V t$ (not reported in the Tables) even from a likelihood point of view. In other words, it is precisely the conditioning on the periodic volatility effects that raises the estimate of $\nu$, and therefore makes the $P S V t$ less attractive than $S V t$ from a likelihood point of view.

An examination of the ranking of the Gaussian PSV models leads to three interesting conclusions. First, all of them dominate the plain vanilla $S V$. Second, the low-dimensional $P S V_{L}$ dominates the high-dimensional $P S V_{C}$. Hence from a likelihood point of view, it is best to explicitly model only the periodic volatility effects that are clearly statistically significant. Third, the $P S V_{C}$ and $P S V_{H}$ are equally ranked. Therefore, even though a full account of

\footnotetext{
${ }^{15}$ Recall that each seasonal effect changes the mean of the return and log-variance equations in one direction, i.e. Monday raises volatility, while Wednesday lowers it. Hence this is roughly equivalent to one-sided hypothesis testing at the $\alpha=5 \%$ level.
} 
seasonality $\left(P S V_{C}\right)$ results in higher log-likelihood than just conditioning on the holiday effect $\left(P S V_{H}\right)$, the penalty on the high dimension of $P S V_{C}$ for lack of parsimony cancels out its likelihood advantage. Figure 5 displays the difference in the period-by-period predictive logdensities between $S V t$ and all periodic specifications over time. The sum of these differences is the difference in the log-likelihoods. Figure 6 shows the cumulative differences, where the final point on these lines is just the log-likelihood. Clearly, the likelihood dominance of $S V t$ can be attributed to accommodating better the recurring outliers in the data.

Tables 8A and 8B present the log-likelihood values for the full sample and for selected days and months. For example, the log-likelihood for Monday is just the sum of the predictive logdensity of each model only for the observations which are Mondays. The ranking of the models does not appear to be reversed in isolating the likelihood over a given seasonal effect. The one exception is the strong $H O L$ effect, for which all $P S V$ and $P S V t$ specifications (all of which condition on at least $H O L$ ) dominate the $S V t$. This is further evidence of the importance of explicitly modelling the holiday effect, even from a likelihood point of view. The likelihood values are consistent with the Bayes factor result that $P S V_{L}$ is the best Gaussian model.

The in-sample conditional dynamics of all $S V$ models are assessed by examining deviations of the density forecasts $\left\{n_{t+1}\right\}$ from the $N(0,1)$ distribution. Table $8 \mathrm{C}$ presents the results. All $P S V$ models perform better than the plain vanilla $S V$. The low-dimensional $P S V_{L}$ and $P S V t_{L}$ are the best among all periodic Gaussian and Student- $t$ models, respectively, in reducing the kurtosis of the density forecasts. Again, $S V t$ is the best performer and reduces substantially the excess kurtosis of the $\left\{n_{t+1}\right\}$ from the $N(0,1)$ distribution. A visually more attractive way to assess the performance of all Gaussian and Student- $t$ models is by simply inspecting the $Q Q$ plots presented in Figures 7 and 8.

Additionally, we perform an out-of-sample exercise using two full years of return data, the years 2002 and 2003, by conditioning on the in-sample parameter estimates for the years 1980-2001, which have been presented in Tables 3-7. Unfortunately, it is not numerically feasible to update the posterior estimate of the parameters every day a new out-of-sample observation is added because of the substantial time it takes to re-run the MCMC algorithm. Further, in the out-of-sample case, it is not possible to compute Bayes factors because the out-of-sample posterior density $\pi(\theta \mid y)$ is not available analytically. The posterior density can only be simulated in-sample using the MCMC estimation algorithm and implementing the techniques of reduced conditional runs of Chib (1995) and Chib and Jeliazkov (2001). Hence we rank the out-of-sample performance of the SV models using the estimate of the out-of-sample likelihoods presented in Tables 9A for 2003 and 9B for 2002-2003. For 2002, $S V t$ is still slightly better in terms of its log-likelihood and all other models are virtually indistinguishable. However, an interesting exception to this rule is that for the 2002-2003 period, the $P S V t$ models have higher likelihood that $S V t$. Finally, the out-of-sample density forecasts are worse than the in-sample values, especially in that the variance of $\left\{n_{t+1}\right\}$ is far from unity and closer to the value of 2 . 


\section{Concluding Remarks}

This paper has provided a comprehensive analysis of the day of the week, holiday, and month of the year seasonal effects in the daily returns and volatility of the S\&P 500 index over the years 1980-2001. We find that Mondays tend to have the lowest return and highest volatility. Wednesdays have the second highest return and the lowest volatility. January has the highest return, while October has the highest volatility. Finally, the first trading weekday after a holiday has a negative average return and very high volatility. More importantly, by bootstrapping the means of daily returns and daily absolute returns, we have demonstrated that these three prominent calendar effects are statistically significant only in daily volatility and not in daily average returns. This result is robust to the exclusion of the three most volatile days of the sample, Monday October 19, 1987 to Wednesday, October 21, 1987. Therefore, the evidence clearly indicates that a stochastic volatility model should perform better if it was generalized to account for the observed seasonal periodicity in volatility.

In this context, the main contribution of the paper is that it introduces and estimates the periodic stochastic volatility model, and then formally examines the interaction between seasonal heteroscedasticity and fat tails. The model-specific results point towards three sets of conclusions. First, consistent with the bootstrapping evidence, we find that conditioning on the periodic seasonal effects results in estimates for the seasonal coefficients, which are large and statistically different from zero only in the volatility equation, and not in the return equation. We also find strong parsimony gains from reducing the dimension of the PSV model by explicitly modelling only the seasonals that for this dataset are statistically significant. There is, therefore, clear misspecification if we use an SV model to form an estimate of tomorrow's volatility without conditioning on the fact that tomorrow could be (say) a Monday in October. In fact, the holiday effect is by far the strongest seasonal effect in both returns and volatility.

Second, the conditioning on day of the week, holiday, and month of the year effects reduces and even eliminates the need for fat tails. The estimate of the degrees of freedom parameter in the Student- $t$ distribution of all PSVt specifications is substantially higher than the one for $S V t$. In effect, the degrees of freedom estimates are so high that the Student- $t$ distribution behaves exactly like a Gaussian.

Finally, the rankings of all SV specifications according to the likelihood-based Bayes factor diagnostic and the one-step ahead density forecasts favour the simple $S V t$ model exclusively because it has the lowest degrees of freedom. All the results above are not sensitive to the Crash of 1987 outliers. 


\begin{tabular}{|cccc|}
\hline \multicolumn{4}{|c}{ Table 1: Descriptive Statistics for the Daily S\&P 500 } \\
Jan 1, 1980 to Dec $31,2001(\mathrm{~T}=5552)$
\end{tabular}

Table 1. Descriptive statistics 


\begin{tabular}{|c|c|c|c|c|c|}
\hline \multicolumn{6}{|c|}{ Table 2A: Day of the Week Effect } \\
\hline & Days & $\begin{array}{l}\text { Mean } \\
\text { of } y_{t}\end{array}$ & $\begin{array}{l}\text { Mean } \\
\text { of } y_{t}^{D C}\end{array}$ & $\begin{array}{l}\text { Mean } \\
\text { of }\left|y_{t}\right|\end{array}$ & $\begin{array}{c}\text { Mean } \\
\text { of }\left|y_{t}^{D C}\right|\end{array}$ \\
\hline Full Sample & 5552 & $\begin{array}{cc}\mathbf{0 . 0 4 3} \\
(0.020, \quad 0.066) \\
\end{array}$ & & $\begin{array}{cc}0.713 \\
(0.697, \quad 0.730) \\
\end{array}$ & \\
\hline$\overline{M O N}$ & 1060 & $\begin{array}{c}0.0009 \\
(-0.066, \quad 0.063)\end{array}$ & $\begin{array}{c}0.052 \\
(0.028, \quad 0.077)\end{array}$ & $\begin{array}{c}\mathbf{0 . 7 6 3} \\
(0.713, \quad 0.818)\end{array}$ & $\begin{array}{c}0.702 \\
(0.685, \quad 0.719)\end{array}$ \\
\hline$T U E$ & 1135 & 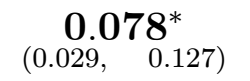 & $\begin{array}{c}0.034 \\
(0.008, \quad 0.059)\end{array}$ & $\begin{array}{c}0.725 \\
(0.692, \quad 0.758)\end{array}$ & $\begin{array}{c}0.711 \\
(0.692, \quad 0.730)\end{array}$ \\
\hline$W E D$ & 1136 & $\begin{array}{c}\mathbf{0 . 0 7 6}^{*} \\
(0.032, \quad 0.121)\end{array}$ & $\begin{array}{c}0.034 \\
(0.008, \quad 0.060)\end{array}$ & $\begin{array}{c}\mathbf{0 . 6 6 2}^{\text {****}} \\
(0.631,, 0.694)\end{array}$ & $\begin{array}{c}0.727 \\
(0.708, \quad 0.747)\end{array}$ \\
\hline$T H U$ & 1114 & $\begin{array}{c}0.014 \\
(-0.033, \quad 0.063)\end{array}$ & $\begin{array}{c}0.050 \\
(0.023, \quad 0.075)\end{array}$ & $\begin{array}{cc}0.708 \\
(0.675, \quad 0.742)\end{array}$ & $\begin{array}{c}0.715 \\
(0.697, \quad 0.734)\end{array}$ \\
\hline$F R I$ & 1107 & $\begin{array}{c}0.041 \\
(-0.008, \quad 0.091) \\
\end{array}$ & $\begin{array}{c}0.043 \\
(0.017, \quad 0.069) \\
\end{array}$ & $\begin{array}{c}0.713 \\
(0.679, \quad 0.749) \\
\end{array}$ & $\begin{array}{c}0.714 \\
(0.695, \quad 0.733) \\
\end{array}$ \\
\hline$\overline{H O L}$ & 184 & $\begin{array}{c}-0.014 \\
(-0.163, \quad 0.130)\end{array}$ & $\begin{array}{c}0.045 \\
(0.021, \quad 0.067)\end{array}$ & $\begin{array}{c}\mathbf{0 . 8 8 2}^{* * *} \\
(0.780, \quad 0.984)\end{array}$ & $\begin{array}{cc}0.708 \\
(0.691, \quad 0.725)\end{array}$ \\
\hline
\end{tabular}

Table 2A: Day of the Week Seasonal Level Effects for the daily returns $y_{t}$, the daily absolute returns $\left|y_{t}\right|$, and their complements.

The variables $y_{t}^{D C}$ and $\left|y_{t}^{D C}\right|$ are the day complements of $y_{t}$ and $\left|y_{t}\right|$ respectively. In other words, they contain the full sample except for the day in that row. For example, the $y_{t}^{D C}$ of Monday are the returns of all days except Monday. HOL captures the non-trading day (post-holiday) effect and is defined as a weekday that follows a weekday for which the market was closed. The numbers in parenthesis are the $5 \%$ and $95 \%$ quantiles for the means generated by 10,000 bootstrap samples. We display the central $90 \%$ of the bootstrap distribution because this leaves $5 \%$ on the side for which the one-sided hypothesis test is performed. In the mean of $y_{t}$ column, a number in bold indicates that the average return for the day in that row is significantly different from zero. In the mean of $\left|y_{t}\right|$ column, a bold number indicates that the central $90 \%$ of the bootstrap distribution of this day is completely non-overlapping to that of its day complement for up to two decimal digits. One asterisk indicates that the relevant one-sided null hypothesis that $y_{t}=y_{t}^{M C}$ is rejected at significance level $\alpha=10 \%$ using bootstrapping. Two asterisks are for rejections at $\alpha=5 \%$, and three asterisks are for rejecting the null hypothesis at $\alpha=1 \%$ significance. 


\begin{tabular}{|c|c|c|c|c|c|}
\hline \multicolumn{6}{|c|}{ Table 2B: Month of the Year Effect } \\
\hline & Days & $\begin{array}{l}\text { Mean } \\
\text { of } y_{t}\end{array}$ & $\begin{array}{c}\text { Mean } \\
\text { of } y_{t}^{M C} \\
\end{array}$ & $\begin{array}{l}\text { Mean } \\
\text { of }\left|y_{t}\right|\end{array}$ & $\begin{array}{c}\text { Mean } \\
\text { of }\left|y_{t}^{M C}\right|\end{array}$ \\
\hline Full Sample & 5552 & $\begin{array}{cc}\mathbf{0 . 0 4 3} \\
(0.020, \quad 0.066) \\
\end{array}$ & & $\begin{array}{cc}0.713 \\
(0.697, \quad 0.730) \\
\end{array}$ & \\
\hline$J A N$ & 462 & $\begin{array}{c}\mathbf{0 . 0 9 9} \\
(0.015, \quad 0.181)\end{array}$ & $\begin{array}{c}0.037 \\
(0.014, \quad 0.061)\end{array}$ & $\begin{array}{c}0.754 \\
(0.697, \quad 0.813)\end{array}$ & $\begin{array}{c}0.710 \\
(0.693, \quad 0.727)\end{array}$ \\
\hline$F E B$ & 422 & $\begin{array}{c}0.019 \\
(-0.055, \quad 0.093)\end{array}$ & $\begin{array}{c}0.045 \\
(0.020, \quad 0.069)\end{array}$ & $\begin{array}{c}0.713 \\
(0.664, \quad 0.777)\end{array}$ & $\begin{array}{c}0.714 \\
(0.696, \quad 0.731)\end{array}$ \\
\hline$M A R$ & 481 & $\begin{array}{c}0.026 \\
(-0.048, \quad 0.099)\end{array}$ & $\begin{array}{c}0.044 \\
(0.049, \quad 0.068)\end{array}$ & $\begin{array}{c}0.707 \\
(0.658, \quad 0.759)\end{array}$ & $\begin{array}{c}0.744 \\
(0.697, \quad 0.732)\end{array}$ \\
\hline$A P R$ & 453 & $\begin{array}{c}0.074 \\
(-0.008, \quad 0.155)\end{array}$ & $\begin{array}{c}0.040 \\
(0.016, \quad 0.063)\end{array}$ & $\begin{array}{c}0.746 \\
(0.691, \quad 0.805)\end{array}$ & $\begin{array}{c}0.711 \\
(0.693, \quad 0.729)\end{array}$ \\
\hline$M A Y$ & 465 & $\begin{array}{c}0.063 \\
(-0.004, \quad 0.126)\end{array}$ & $\begin{array}{c}0.041 \\
(0.016, \quad 0.065)\end{array}$ & $\begin{array}{c}\mathbf{0 . 6 5 5 0 * *} \\
(0.607, \quad 0.693)\end{array}$ & $\begin{array}{c}0.719 \\
(0.702, \quad 0.737)\end{array}$ \\
\hline$J U N E$ & 472 & $\begin{array}{c}0.044 \\
(-0.016, \quad 0.106)\end{array}$ & $\begin{array}{c}0.042 \\
(0.017, \quad 0.067)\end{array}$ & $\begin{array}{c}\mathbf{0 . 6 2 0} \mathbf{6}^{* * *} \\
(0.583, \quad 0.658)\end{array}$ & $\begin{array}{c}0.722 \\
(0.704, \quad 0.741)\end{array}$ \\
\hline$J U L Y$ & 466 & $\begin{array}{c}0.030 \\
(-0.033, \quad 0.092)\end{array}$ & $\begin{array}{c}0.044 \\
(0.019, \quad 0.068)\end{array}$ & $\begin{array}{c}\mathbf{0 . 6 4 4}^{\text {****}} \\
(0.605, \quad 0.684)\end{array}$ & $\begin{array}{c}0.720 \\
(0.703, \quad 0.738)\end{array}$ \\
\hline$A U G$ & 486 & $\begin{array}{c}-0.003 \\
(-0.079, \quad 0.071)\end{array}$ & $\begin{array}{c}0.047 \\
(0.022, \quad 0.071)\end{array}$ & $\begin{array}{c}0.697 \\
(0.644, \quad 0.752)\end{array}$ & $\begin{array}{c}0.715 \\
(0.698, \quad 0.733)\end{array}$ \\
\hline$S E P T$ & 445 & $\begin{array}{c}-0.044^{* *} \\
(-0.127, \quad 0.036)\end{array}$ & $\begin{array}{c}0.050 \\
(0.026, \quad 0.074)\end{array}$ & $\begin{array}{c}0.728 \\
(0.672, \quad 0.789)\end{array}$ & $\begin{array}{c}0.712 \\
(0.695, \quad 0.730)\end{array}$ \\
\hline$O C T$ & 486 & $\begin{array}{c}0.038 \\
(-0.093, \quad 0.161)\end{array}$ & $\begin{array}{c}0.043 \\
(0.021, \quad 0.065)\end{array}$ & $\begin{array}{c}\mathbf{0 . 9 0 2}^{\text {****}} \\
(0.802, \quad 1.018)\end{array}$ & $\begin{array}{c}0.695 \\
(0.681, \quad 0.711)\end{array}$ \\
\hline NOV & 448 & $\begin{array}{c}\mathbf{0 . 0 8 3} \\
(0.010, \quad 0.158)\end{array}$ & $\begin{array}{c}0.039 \\
(0.016, \quad 0.063)\end{array}$ & $\begin{array}{c}0.726 \\
(0.677, \quad 0.777)\end{array}$ & $\begin{array}{c}0.712 \\
(0.695, \quad 0.731)\end{array}$ \\
\hline$D E C$ & 466 & $\begin{array}{c}\mathbf{0 . 0 8 2} \\
(0.011, \quad 0.151)\end{array}$ & $\begin{array}{c}0.039 \\
(0.014, \quad 0.063)\end{array}$ & $\begin{array}{c}0.671^{*} \\
(0.624, \quad 0.719)\end{array}$ & $\begin{array}{c}0.717 \\
(0.700, \quad 0.735)\end{array}$ \\
\hline
\end{tabular}

Table 2B: Month of the Year Seasonal Level Effects for the daily returns $y_{t}$, the daily absolute returns $\left|y_{t}\right|$, and their complements.

The variables $y_{t}^{M C}$ and $\left|y_{t}^{M C}\right|$ are the month complements of $y_{t}$ and $\left|y_{t}\right|$ respectively. In other words, they contain the full sample except for the month in that row. For example, the $y_{t}^{M C}$ of January are the returns of all days except for January. The numbers in parenthesis are the $5 \%$ and $95 \%$ quantiles for the means generated by 10,000 bootstrap samples. In the mean of $y_{t}$ column, a number in bold indicates that the average return for the month in that row is significantly different from zero. In the mean of $\left|y_{t}\right|$ column, a bold number indicates that the central $90 \%$ of the bootstrap distribution of this month is completely non-overlapping to that of its month complement for up to two decimal digits. One asterisk indicates that the relevant one-sided null hypothesis that $y_{t}=y_{t}^{M C}$ is rejected at significance level $\alpha=10 \%$ using bootstrapping. Two asterisks are for rejections at $\alpha=5 \%$, and three asterisks are for rejecting the null hypothesis at $\alpha=1 \%$ significance. 


\begin{tabular}{|c|c|c|c|c|}
\hline \multicolumn{5}{|c|}{$\begin{array}{r}\text { Table } 2 \mathrm{C}: \\
\text { Seasonality with and without the three }\end{array}$} \\
\hline & \multicolumn{2}{|c|}{ Mean of $y_{t}$} & \multicolumn{2}{|c|}{ Mean of $\left|y_{t}\right|$} \\
\hline & FULL SAMPLE & FULL - 3 & FULL SAMPLE & FULL - 3 \\
\hline ALL DAYS & $\begin{array}{c}0.043 \\
(0.020, \quad 0.066)\end{array}$ & $\begin{array}{c}0.044 \\
(0.023, \quad 0.066)\end{array}$ & $\begin{array}{c}0.713 \\
(0.697, \quad 0.730)\end{array}$ & $\begin{array}{c}0.707 \\
(0.692 . \quad 0.723)\end{array}$ \\
\hline$M O N$ & $\begin{array}{cc}0.0009 \\
(-0.066, \quad 0.063)\end{array}$ & $\begin{array}{c}0.022 \\
(-0.032, \quad 0.077)\end{array}$ & $\begin{array}{c}0.763^{* *} \\
(0.713, \quad 0.818)\end{array}$ & $\begin{array}{c}0.742^{*} \\
(0.703, \quad 0.781)\end{array}$ \\
\hline$T U E$ & $\begin{array}{c}0.078^{*} \\
(0.029, \quad 0.127)\end{array}$ & $\begin{array}{c}0.073 \\
(0.025, \quad 0.121)\end{array}$ & $\begin{array}{c}0.725 \\
(0.692, \quad 0.758)\end{array}$ & $\begin{array}{c}0.721 \\
(0.689, \quad 0.754)\end{array}$ \\
\hline$W E D$ & $\begin{array}{cc}0.076^{*} \\
(0.032, \quad 0.121)\end{array}$ & $\begin{array}{c}0.068 \\
(0.025, \quad 0.112) \\
\end{array}$ & $\begin{array}{c}0.662^{* * *} \\
(0.631, \quad 0.694)\end{array}$ & $\begin{array}{c}0.654^{* * *} \\
(0.625, \quad 0.684)\end{array}$ \\
\hline$O C T$ & $\begin{array}{c}0.038 \\
(-0.093, \quad 0.161)\end{array}$ & $\begin{array}{cc}0.057 \\
(-0.039, \quad 0.151)\end{array}$ & $\begin{array}{c}0.902^{* * *} \\
(0.802, \quad 1.018)\end{array}$ & $\begin{array}{c}0.831^{* * *} \\
(0.761, \quad 0.905) \\
\end{array}$ \\
\hline
\end{tabular}

Table 2C. Seasonality with and without the three most volatile days of the sample period: Monday October 19, 1987 to Wednesday, October 21, 1987.

The numbers in parenthesis are the 5\% and $95 \%$ quantiles for the means generated by 10,000 bootstrap samples. One asterisk indicates that the relevant one-sided null hypothesis that $y_{t}=y_{t}^{M C}$ is rejected at significance level $\alpha=10 \%$ using bootstrapping. Two asterisks are for rejections at $\alpha=5 \%$, and three asterisks are for rejecting the null hypothesis at $\alpha=1 \%$ significance. 


\begin{tabular}{|c|c|c|c|c|}
\hline \multirow{2}{*}{\multicolumn{5}{|c|}{\begin{tabular}{|c|} 
Table 2D: \\
Statistical Significance in Volatility by Subsamples
\end{tabular}}} \\
\hline & & & & \\
\hline & $1980-84$ & $1985-89$ & 1990-94 & 1995-2001 \\
\hline ALL DAYS & \begin{tabular}{|l|l|}
0.730 \\
\end{tabular} & 0.727 & 0.553 & \begin{tabular}{|l|l|}
0.813 \\
\end{tabular} \\
\hline$M O N$ & $0.822^{* * *}$ & 0.822 & $0.605^{*}$ & 0.792 \\
\hline$W E D$ & 0.722 & $0.653^{*}$ & $0.472^{* * *}$ & $0.759^{*}$ \\
\hline$\overline{H O L}$ & 0.835 & $0.937^{*}$ & $0.731^{* *}$ & 0.972 \\
\hline$J A N$ & 0.727 & 0.833 & 0.600 & \begin{tabular}{|l|l|}
0.829 \\
\end{tabular} \\
\hline$M A Y$ & $0.576^{* * *}$ & $0.637^{*}$ & 0.528 & 0.797 \\
\hline$J U N E$ & $0.637^{* *}$ & $0.633^{* *}$ & 0.519 & $0.673^{* * *}$ \\
\hline$J U L Y$ & $0.626^{* *}$ & $0.631^{* *}$ & $0.489^{* *}$ & 0.776 \\
\hline$O C T$ & 0.791 & $1.23^{* *}$ & 0.617 & $0.947^{* *}$ \\
\hline
\end{tabular}

Table 2D: The statistical significance of selected seasonal effects in volatility for four subsamples.

The first three subsamples are of five years each and the last is of the remaining seven years. The displayed seasonal variables are the seven calendar effects (two days, four months, one holiday), which in the full sample (1980-2001) were statistically significant in volatility with at least $95 \%$ confidence, plus January. 


\begin{tabular}{|c||c||cc|cc|c|}
\hline \multicolumn{7}{|c|}{ Table 3: $S V$ Parameter Estimates } \\
\hline $\begin{array}{c}S V \\
\text { Parameter }\end{array}$ & $\begin{array}{c}\text { Posterior } \\
\text { Mean }\end{array}$ & $\begin{array}{c}\text { MCMC } \\
\text { Std Dev }\end{array}$ & NSE & Lower & Upper & RNI \\
\hline \hline$\alpha$ & 0.039 & 0.010 & 0.0002 & 0.022 & 0.055 & 1.78 \\
$\rho$ & 0.052 & 0.014 & 0.0002 & 0.029 & 0.075 & 1.28 \\
\hline \hline$\mu$ & -0.197 & 0.078 & 0.0022 & -0.326 & -0.069 & 4.13 \\
$\beta_{1}$ & -0.088 & 0.010 & 0.0004 & -0.105 & -0.072 & 6.87 \\
$\phi$ & 0.969 & 0.005 & 0.0001 & 0.961 & 0.977 & 3.95 \\
$\sigma^{2}$ & 0.028 & 0.004 & 0.0002 & 0.022 & 0.036 & 6.08 \\
\hline
\end{tabular}

Table 3. SV Model:

MCMC Parameter Estimates for the Full Sample.

In all specifications, the MCMC chain run for 5,000 iterations after an initial burn-in of 1,000 iterations. Lower and Upper indicate the $5 \%$ and $95 \%$ percentiles of the MCMC draws. 


\begin{tabular}{|ccccccc|}
\hline \multicolumn{7}{|c|}{ Table 4A: } \\
SV Estimates of $\nu$ for all models, Full Sample \\
\hline Model & $\begin{array}{c}\text { Posterior } \\
\text { Mean }\end{array}$ & $\begin{array}{c}\text { MCMC } \\
\text { Std Dev }\end{array}$ & NSE & Lower & Upper & RNI \\
\hline \hline$S V t$ & 11.11 & 1.95 & 0.229 & 8.28 & 16.12 & 68.96 \\
\hline \hline$P S V t_{C}$ & 52.3 & 11.3 & 2.89 & 27.8 & 65.6 & 327.6 \\
$P S V t_{L}$ & 46.7 & 11.7 & 2.94 & 28.8 & 66.2 & 315.7 \\
$P S V t_{H}$ & 37.9 & 9.43 & 2.00 & 23.7 & 60.2 & 14.41 \\
\hline
\end{tabular}

\begin{tabular}{|ccccccc|}
\hline \multicolumn{7}{|c|}{ Table 4B: } \\
Estimates of $\nu$ excluding October 19-21, 1987 \\
\hline Model & $\begin{array}{c}\text { Posterior } \\
\text { Mean }\end{array}$ & $\begin{array}{c}\text { MCMC } \\
\text { Std Dev }\end{array}$ & NSE & Lower & Upper & RNI \\
\hline \hline$S V t$ & 11.98 & 1.88 & 0.200 & 9.35 & 15.25 & 56.41 \\
\hline \hline$P S V t_{C}$ & 55.9 & 5.07 & 1.15 & 47.9 & 64.4 & 256.4 \\
$P S V t_{L}$ & 58.3 & 6.38 & 1.53 & 47.0 & 65.2 & 289.1 \\
$P S V t_{H}$ & 46.5 & 15.2 & 3.98 & 19.1 & 63.9 & 343.4 \\
\hline
\end{tabular}

Table 4. MCMC estimates of the degrees of freedom parameter $\nu$ for all fat-tailed $S V t$ specifications with and without seasonal periodicity. 


\begin{tabular}{|c|c||c||cc|cc|c|}
\hline \multicolumn{7}{|c|}{ Table 5A: $P S V_{C}$ Seasonal Parameters in the Mean } \\
\hline $\begin{array}{c}\text { Seasonal } \\
\text { Effects }\end{array}$ & $\begin{array}{c}P S V_{C} \\
\text { Parameter }\end{array}$ & $\begin{array}{c}\text { Posterior } \\
\text { Mean }\end{array}$ & $\begin{array}{c}\text { MCMC } \\
\text { Std Dev }\end{array}$ & NSE & Lower & Upper & RNI \\
\hline \hline const & $\delta_{0}$ & 0.059 & 0.024 & 0.0004 & 0.020 & 0.098 & 1.21 \\
MON & $\delta_{1}$ & -0.018 & 0.035 & 0.0005 & -0.077 & 0.038 & 1.10 \\
TUE & $\delta_{2}$ & -0.024 & 0.033 & 0.0004 & -0.078 & 0.032 & 0.76 \\
WED & $\delta_{3}$ & 0.002 & 0.032 & 0.0006 & -0.051 & 0.055 & 1.52 \\
THU & $\delta_{4}$ & -0.043 & 0.033 & 0.0004 & -0.097 & 0.011 & 0.86 \\
\hline \hline HOL & $\delta_{5}$ & -0.089 & 0.073 & 0.0011 & -0.209 & 0.032 & 1.17 \\
\hline \hline Fourier 1 & $\delta_{6}$ & 0.011 & 0.015 & 0.0003 & -0.014 & 0.036 & 1.60 \\
Fourier 2 & $\delta_{7}$ & 0.013 & 0.015 & 0.0002 & -0.011 & 0.037 & 1.13 \\
Fourier 3 & $\delta_{8}$ & -0.006 & 0.015 & 0.0002 & -0.030 & 0.018 & 0.89 \\
Fourier 4 & $\delta_{9}$ & 0.015 & 0.014 & 0.0002 & -0.009 & 0.039 & 0.80 \\
Fourier5 & $\delta_{10}$ & -0.008 & 0.015 & 0.0003 & -0.032 & 0.017 & 1.54 \\
Fourier6 & $\delta_{11}$ & 0.002 & 0.014 & 0.0002 & -0.021 & 0.026 & 1.09 \\
\hline
\end{tabular}

\begin{tabular}{|c|c|c|c|c|c|c|c|}
\hline \multicolumn{8}{|c|}{ Table 5B: $P S V_{C}$ Seasonal Parameters in the log-Variance } \\
\hline $\begin{array}{c}\text { Seasonal } \\
\text { Effect }\end{array}$ & $\begin{array}{c}P S V_{C} \\
\text { Parameter }\end{array}$ & $\begin{array}{c}\text { Posterior } \\
\text { Mean }\end{array}$ & $\begin{array}{l}\text { MCMC } \\
\text { Std Dev }\end{array}$ & $\mathrm{NSE}$ & Lower & Upper & RNI \\
\hline const & $\mu$ & -0.200 & 0.088 & 0.0047 & -0.346 & -0.052 & 14.33 \\
\hline$M O N$ & $\gamma_{1}$ & 0.082 & 0.064 & 0.0040 & -0.025 & 0.187 & 19.68 \\
\hline$T U E$ & $\gamma_{2}$ & -0.014 & 0.065 & 0.0044 & -0.124 & 0.090 & 23.70 \\
\hline$W E D$ & $\gamma_{3}$ & -0.131 & 0.062 & 0.0046 & -0.234 & -0.029 & 27.10 \\
\hline$T H U$ & $\gamma_{4}$ & -0.020 & 0.063 & 0.0039 & -0.123 & 0.081 & 19.59 \\
\hline$H O L$ & $\gamma_{5}$ & 0.415 & 0.111 & 0.0037 & 0.233 & 0.601 & 5.59 \\
\hline Fourier 1 & $\gamma_{6}$ & 0.151 & 0.085 & 0.0090 & 0.013 & 0.293 & $\overline{55.43}$ \\
\hline Fourier 2 & $\gamma_{7}$ & 0.050 & 0.080 & 0.0076 & -0.087 & 0.175 & 44.42 \\
\hline Fourier3 & $\gamma_{8}$ & 0.006 & 0.054 & 0.0040 & -0.084 & 0.094 & 27.33 \\
\hline Fourier4 & $\gamma_{9}$ & -0.064 & 0.051 & 0.0029 & -0.150 & 0.021 & 15.34 \\
\hline Fourier5 & $\gamma_{10}$ & 0.039 & 0.042 & 0.0018 & -0.027 & 0.109 & 9.51 \\
\hline Fourier6 & $\gamma_{11}$ & -0.069 & 0.042 & 0.0018 & -0.137 & -0.002 & 9.34 \\
\hline
\end{tabular}

Table 5. $P S V_{C}$ Model:

MCMC Estimates of the Seasonal Parameters in the Conditional Mean and log-Variance for the Full Sample. 


\begin{tabular}{|c|c||c||cc|cc|c|}
\hline \multicolumn{7}{|c|}{ Table 6A: } & $P V_{L}$ Seasonal Parameters in the Mean \\
\hline $\begin{array}{c}\text { Seasonal } \\
\text { Effect }\end{array}$ & $\begin{array}{c}P S V_{L} \\
\text { Parameter }\end{array}$ & $\begin{array}{c}\text { Posterior } \\
\text { Mean }\end{array}$ & $\begin{array}{c}\text { MCMC } \\
\text { Std Dev }\end{array}$ & NSE & Lower & Upper & RNI \\
\hline \hline MON & $\delta_{1}$ & 0.008 & 0.029 & 0.0004 & -0.040 & 0.056 & 1.06 \\
$W E D$ & $\delta_{2}$ & 0.025 & 0.026 & 0.0004 & -0.017 & 0.067 & 0.946 \\
$H O L$ & $\delta_{3}$ & -0.081 & 0.073 & 0.0012 & -0.204 & 0.041 & 1.33 \\
\hline \hline$J A N$ & $\delta_{4}$ & 0.053 & 0.040 & 0.0005 & -0.014 & 0.118 & 0.859 \\
OCT & $\delta_{5}$ & 0.014 & 0.039 & 0.0005 & -0.050 & 0.078 & 0.656 \\
\hline
\end{tabular}

\begin{tabular}{|c|c||c||cc|cc|c|}
\hline \multicolumn{7}{|c|}{ Table 6B: $P S V_{L}$ Seasonal Parameters in the log-Variance } \\
\hline $\begin{array}{c}\text { Seasonal } \\
\text { Effect }\end{array}$ & $\begin{array}{c}P S V_{L} \\
\text { Parameter }\end{array}$ & $\begin{array}{c}\text { Posterior } \\
\text { Mean }\end{array}$ & $\begin{array}{c}\text { MCMC } \\
\text { Std Dev }\end{array}$ & NSE & Lower & Upper & RNI \\
\hline \hline$M O N$ & $\gamma_{1}$ & 0.083 & 0.056 & 0.0032 & -0.008 & 0.176 & 16.07 \\
$W E D$ & $\gamma_{2}$ & -0.130 & 0.053 & 0.0025 & -0.216 & -0.043 & 10.86 \\
$H O L$ & $\gamma_{3}$ & 0.433 & 0.112 & 0.0046 & 0.248 & 0.622 & 8.51 \\
\hline \hline$J A N$ & $\gamma_{4}$ & 0.215 & 0.108 & 0.0063 & 0.036 & 0.392 & 16.77 \\
& & & & & & & \\
$O C T$ & $\gamma_{5}$ & 0.224 & 0.112 & 0.0050 & 0.040 & 0.405 & 10.20 \\
\hline
\end{tabular}

Table 6. $P S V_{L}$ Model:

MCMC Estimates of the Seasonal Parameters in the Conditional Mean and log-Variance for the Full Sample.

\begin{tabular}{|c|c||c||cc|cc|c|}
\hline \multicolumn{7}{|c|}{ Table 7: $P S V_{H}$ Holiday Effects } \\
\hline $\begin{array}{c}\text { Seasonal } \\
\text { Effect }\end{array}$ & $\begin{array}{c}P S V_{H} \\
\text { Parameter }\end{array}$ & $\begin{array}{c}\text { Posterior } \\
\text { Mean }\end{array}$ & $\begin{array}{c}\text { MCMC } \\
\text { Std Dev }\end{array}$ & NSE & Lower & Upper & RNI \\
\hline \hline$H O L$ & $\delta_{1}$ & -0.091 & 0.073 & 0.0014 & -0.211 & 0.025 & 1.92 \\
$H O L$ & $\gamma_{1}$ & 0.456 & 0.113 & 0.0040 & 0.272 & 0.646 & 6.28 \\
\hline
\end{tabular}

Table 7. $P S V_{H}$ Model:

MCMC Estimates of the Holiday Effect Parameters in the Conditional Mean and log-Variance for the Full Sample. 


\begin{tabular}{|c|cccccc|}
\hline \multicolumn{7}{|c|}{ Table 8A: In-Sample Log-Likelihood } \\
\hline MODEL & Full Sample & $M O N$ & $W E D$ & $H O L$ & $J A N$ & $O C T$ \\
\hline \hline$S V$ & -8387.8 & -1701.8 & -1569.4 & -337.6 & -952.2 & -898.9 \\
\hline \hline$P S V_{C}$ & -8287.0 & -1657.3 & -1588.3 & -300.1 & -889.2 & -919.1 \\
$P S V_{L}$ & -8140.8 & -1664.3 & -1594.2 & -299.0 & -764.5 & -881.6 \\
$P S V_{H}$ & -8360.6 & -1688.7 & -1568.0 & -305.5 & -933.8 & -909.4 \\
\hline \hline$S V t$ & -7765.0 & -1555.4 & -1514.7 & -302.7 & -707.7 & -785.5 \\
\hline$P S V t_{C}$ & -7989.3 & -1615.0 & -1569.0 & -292.4 & -730.9 & -855.8 \\
$P S V t_{L}$ & -7939.3 & -1596.0 & -1562.8 & -293.4 & -726.6 & -817.8 \\
$P S V t_{H}$ & -7932.2 & -1606.8 & -1550.2 & -292.5 & -735.4 & -830.0 \\
\hline
\end{tabular}

\begin{tabular}{|c|cccc|}
\hline \multicolumn{5}{|c|}{ Table 8B: } \\
Log-Likelihood excluding October 19-21, 1987 \\
\hline MODEL & Full Sample & $M O N$ & $W E D$ & $O C T$ \\
\hline \hline$S V$ & -8314.2 & -1635.2 & -1565.8 & -825.3 \\
\hline \hline$P S V_{C}$ & -8213.9 & -1591.1 & -1584.7 & -846.0 \\
$P S V_{L}$ & -8065.5 & -1595.8 & -1590.5 & -806.2 \\
$P S V_{H}$ & -8283.1 & -1618.1 & -1564.4 & -831.9 \\
\hline \hline$S V t$ & -7779.3 & -1536.6 & -1511.1 & -759.8 \\
\hline \hline$P S V t_{C}$ & -7942.3 & -1575.0 & -1565.4 & -808.3 \\
$P S V t_{L}$ & -7900.3 & -1563.8 & -1559.2 & -778.7 \\
$P S V t_{H}$ & -7891.6 & -1573.1 & -1546.6 & -789.3 \\
\hline
\end{tabular}

\begin{tabular}{|ccccc|}
\hline \multicolumn{4}{|c}{ Table 8C: In-Sample $\left\{n_{t+1}\right\}$ Diagnostics } \\
MODEL & VAR & SKEW & KURT & BL(30) \\
\hline \hline$S V$ & 1.26 & -1.63 & 52.9 & 41.0 \\
\hline \hline$P S V_{C}$ & 1.25 & -3.31 & 49.1 & 40.6 \\
$P S V_{L}$ & 1.19 & -5.38 & 40.9 & 45.1 \\
$P S V_{H}$ & 1.26 & -2.36 & 54.4 & 40.4 \\
\hline \hline$S V t$ & 1.11 & -3.23 & 9.47 & 40.7 \\
\hline \hline$P S V t_{C}$ & 1.08 & -2.80 & 29.4 & 49.3 \\
$P S V t_{L}$ & 1.06 & -4.60 & 18.7 & 44.8 \\
$P S V t_{H}$ & 1.05 & -4.86 & 19.9 & 45.3 \\
\hline
\end{tabular}

Table 8. In-Sample Diagnostics

The in-sample period is 1980-2001. Tables 8A and 8B isolate the likelihood of all models for selected days and months. The VAR, SKEW, KURT and BL(30) statistics are for the in-sample one-step ahead density forecasts. $\mathrm{BL}(30)$ is the Box-Ljung statistic at 30 lags. Note that $\chi^{2}(30 ; .90)=40.3$ and $\chi^{2}(30 ; .95)=$ 43.8 . 


\begin{tabular}{|c|ccccc|}
\hline \multicolumn{7}{|c|}{ Table 9A: } \\
Out-of-Sample Diagnostics for 2002 \\
\hline MODEL & log-L & VAR & SKEW & KURT & BL(30) \\
\hline \hline$S V$ & -533.4 & 1.96 & 0.55 & -1.56 & 38.9 \\
\hline \hline$P S V_{C}$ & -539.8 & 2.02 & 0.78 & -1.30 & 43.5 \\
& & & & & \\
$P S V_{L}$ & -533.3 & 2.00 & 0.79 & -1.45 & 38.5 \\
& & & & & \\
$P S V_{H}$ & -533.4 & 1.98 & 0.72 & -1.52 & 38.8 \\
\hline \hline$S V t$ & -501.6 & 1.62 & 0.94 & -2.36 & 41.5 \\
\hline \hline$P S V t_{H}$ & -535.3 & 1.83 & 0.91 & -1.49 & 47.9 \\
& & & & & \\
$P S V t_{L}$ & -527.5 & 1.80 & 0.80 & -1.85 & 44.4 \\
& & & & & \\
$P S V t_{H}$ & -526.5 & 1.78 & 0.84 & -1.88 & 42.8 \\
\hline
\end{tabular}

\begin{tabular}{|c|ccccc|}
\hline \multicolumn{7}{|c|}{ Table 9B: } \\
Out-of-Sample Diagnostics for & 2002-2003 \\
\hline MODEL & log-L & VAR & SKEW & KURT & BL $(30)$ \\
\hline \hline$S V$ & -1066.9 & 1.95 & 0.78 & -2.20 & 74.6 \\
\hline \hline$P S V_{C}$ & -1079.7 & 2.01 & 1.10 & -1.83 & 83.6 \\
& & & & & \\
$P S V_{L}$ & -1066.7 & 2.00 & 1.11 & -2.06 & 73.8 \\
& & & & & \\
$P S V_{H}$ & -1066.8 & 1.98 & 1.02 & -2.15 & 74.5 \\
\hline \hline$S V t$ & -1003.2 & 1.62 & 1.33 & -3.34 & 79.5 \\
\hline \hline$P S V t_{C}$ & -925.8 & 1.57 & 0.17 & -1.60 & 49.2 \\
& & & & & \\
$P S V t_{L}$ & -920.6 & 1.56 & 0.06 & -1.73 & 47.9 \\
& & & & & \\
$P S V t_{H}$ & -921.0 & 1.55 & 0.08 & -1.65 & 48.1 \\
\hline
\end{tabular}

Table 9. Out-of-Sample Diagnostics

The out-of-sample period is 2002 in Table 9A, and 2002-2003 in Table 9B. 


\begin{tabular}{|ccc|}
\hline \multicolumn{3}{|c|}{ Table 10A: Interpreting Bayes Factors ${ }^{16}$} \\
\hline $2 \ln \left(B_{21}\right)$ & $B_{21}$ & Evidence against $M_{1}$ \\
\hline \hline 0 to 2 & 1 to 3 & Not worth more than a bare mention \\
2 to 6 & 3 to 20 & Positive \\
6 to 10 & 20 to 150 & Strong \\
$>10$ & $>150$ & Very strong \\
\hline
\end{tabular}

\begin{tabular}{|cccccccc|}
\hline \multicolumn{7}{|c|}{ Table 10B: Bayes Factors $\left(2 \ln B_{i j}\right)$} \\
\hline MODEL & $P S V_{C}$ & $P S V_{L}$ & $P S V_{H}$ & $S V t$ & $P S V t_{C}$ & $P S V t_{L}$ & $P S V t_{H}$ \\
\hline \hline$S V$ & -43.6 & -433 & -44.7 & -1226 & -672 & -857 & -909 \\
\hline \hline$P S V_{C}$ & & -390 & -1.06 & -1182 & -628 & -813 & -865 \\
$P S V_{L}$ & & & 388 & -793 & -2583 & -2768 & -2820 \\
$P S V_{H}$ & & & -1181 & -627 & -812 & -864 \\
\hline \hline$S V t$ & & & & 554 & 369 & 317 \\
\hline \hline$P S V t_{C}$ & & & & & -185 & -237 \\
$P S V t_{L}$ & & & & & & -52 \\
\hline
\end{tabular}

Table 10. Bayes Factors

The entries are two times the natural of the Bayes factor for the row model versus the column model. We use $2 \ln B_{i j}$ so as to be on the same scale as the likelihood ratio statistics. These are in-sample results for the period 1980-2001. For a more detailed interpretation of these numbers see Section 6.2.

\footnotetext{
${ }^{16}$ See Kass and Raftery (1995).
} 


\section{DAY OF THE WEEK VOLATILITY}

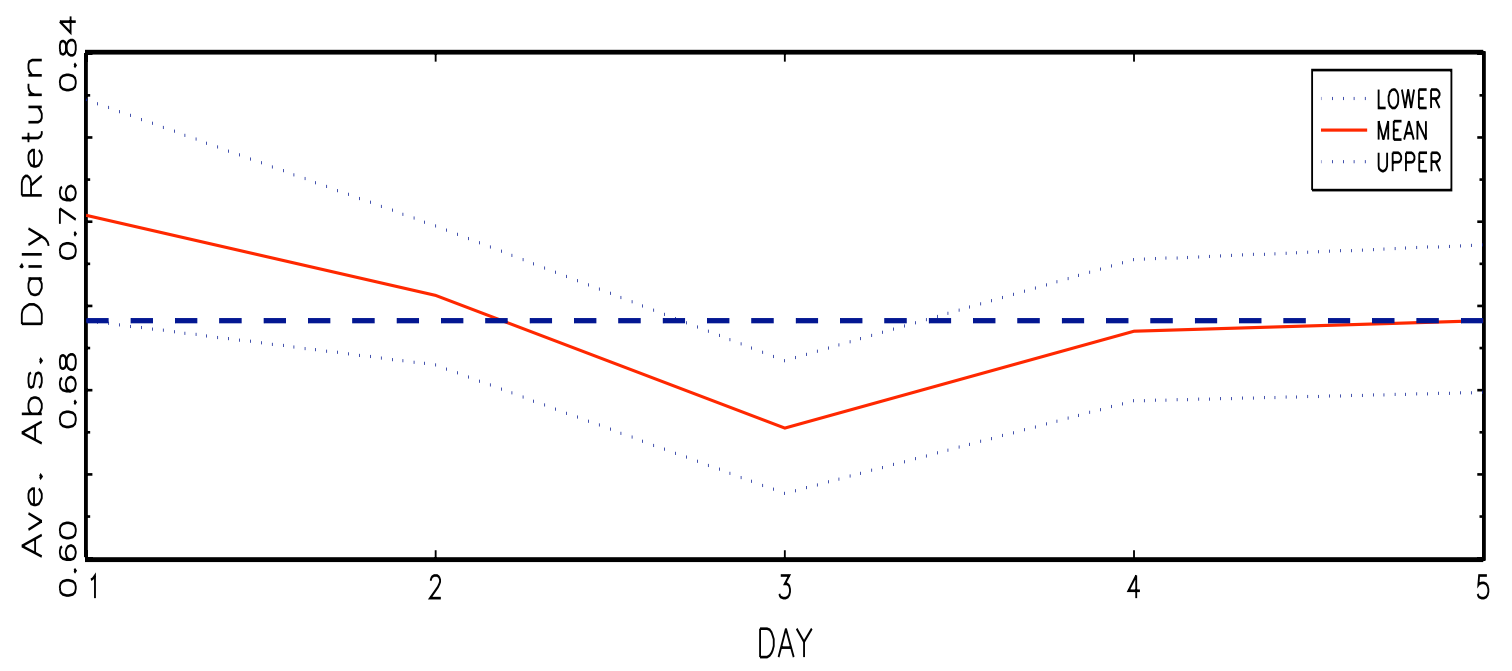

\section{MONTH OF THE YEAR VOLATILITY}

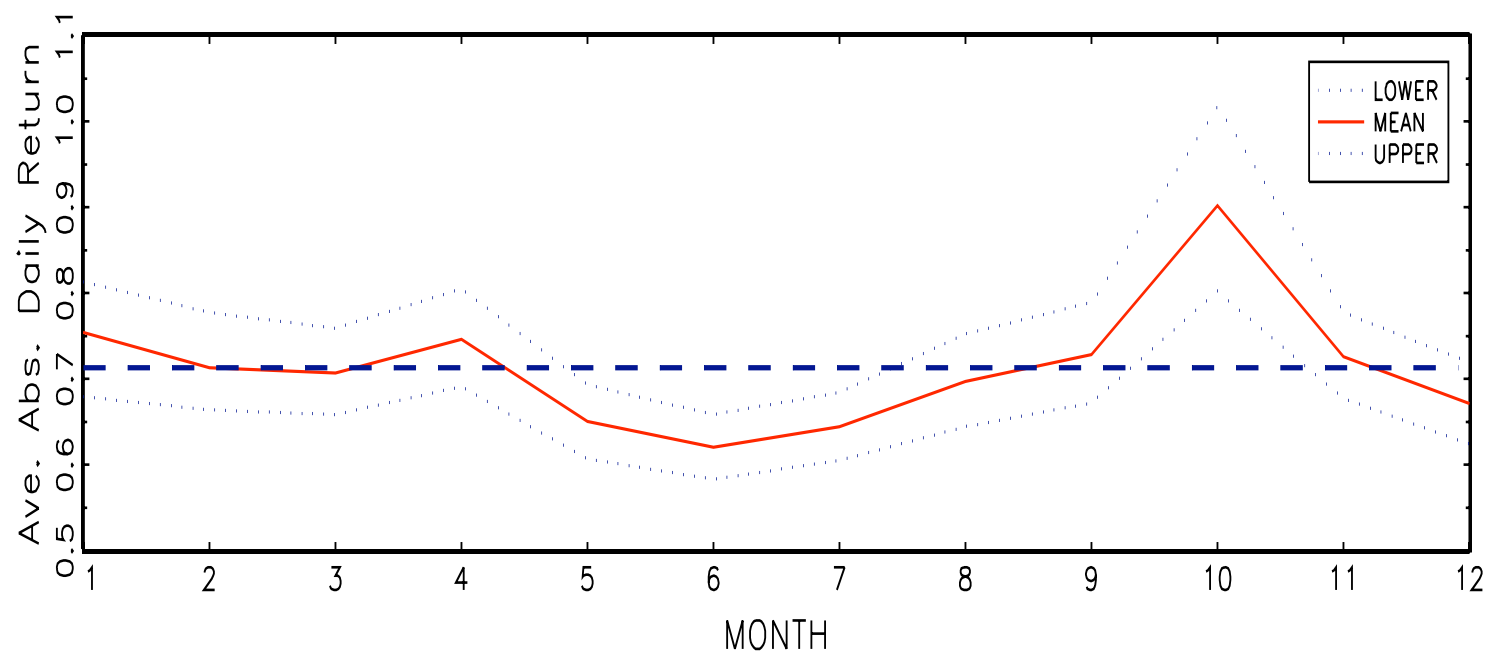

Figure 1: Seasonal Heteroscedasticity. These are the day of the week and month of the year average absolute returns. Lower and Upper indicate the $5 \%$ and $95 \%$ bootstrap confidence intervals, respectively. The horizontal line is the average daily return across all days and months. 
MONDAY

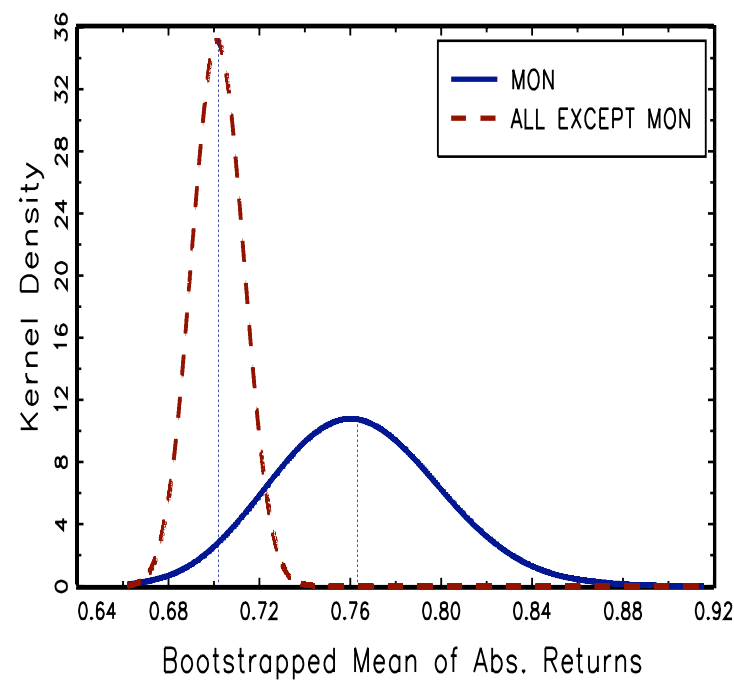

WEDNESDAY

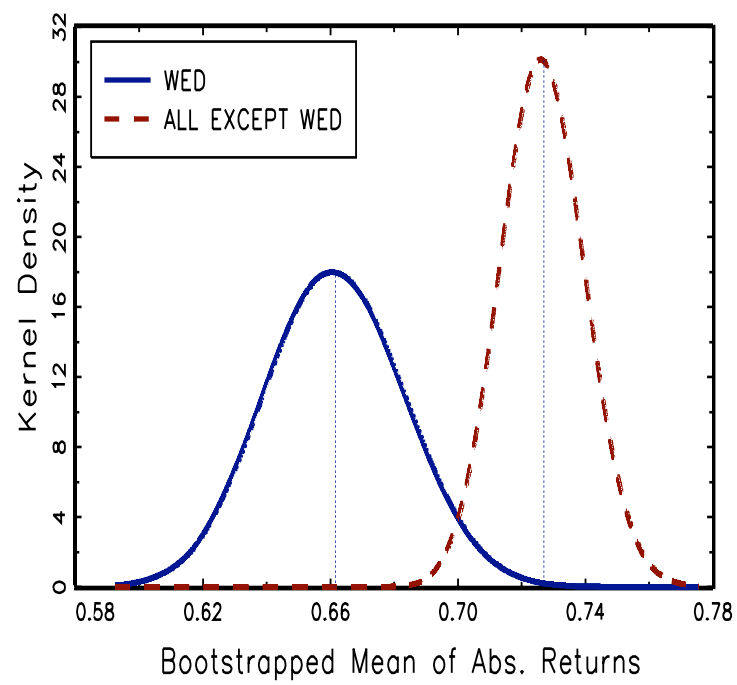

MONDAY EXCLUDING 3 DAYS

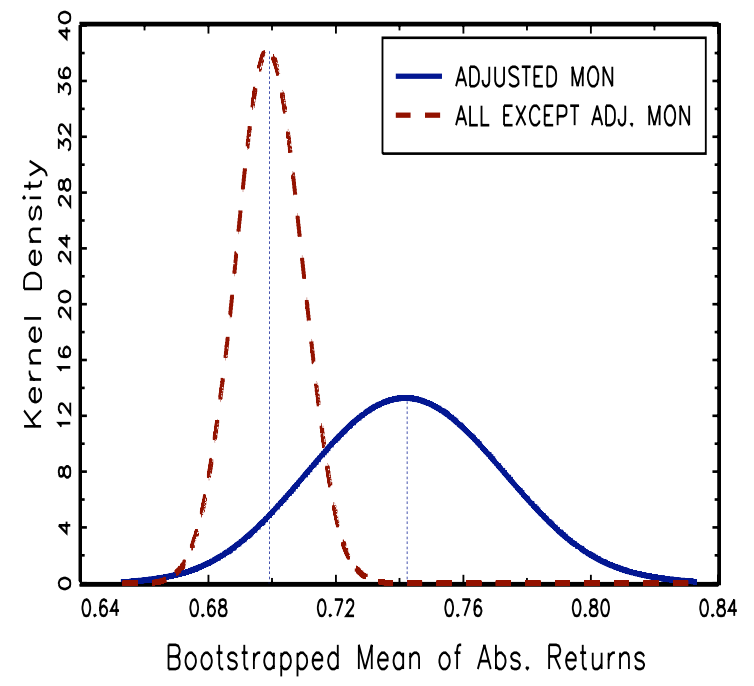

WEDNESDAY EXCLUDING 3 DAYS

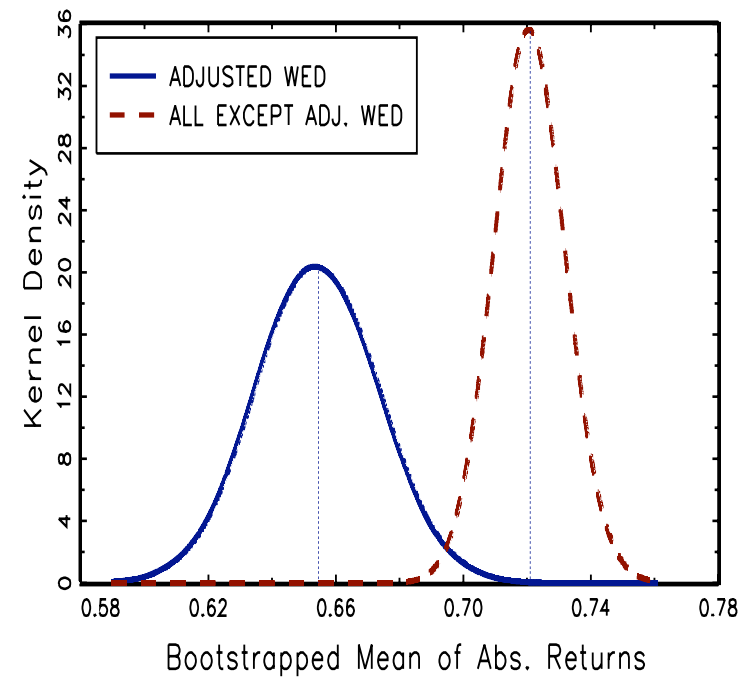

Figure 2: The Monday and Wednesday volatility effects. These are the kernel densities of the means of absolute returns generated by 10,000 bootstrap samples of the original sample. The vertical lines are drawn at the sample means. The adjusted samples exclude the three most volatile days of the sample: Monday, October 19, 1987 to Wednesday, October 21, 1987. 
OCTOBER

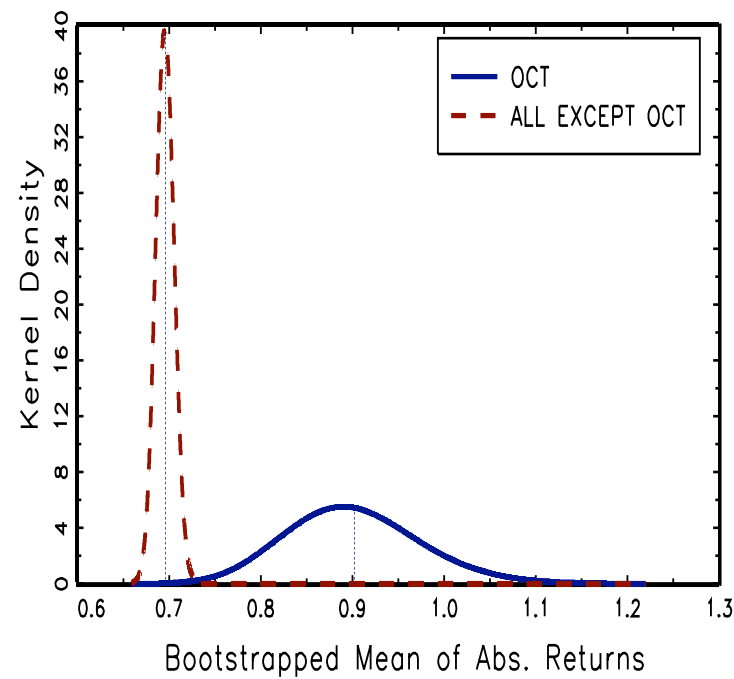

JANUARY

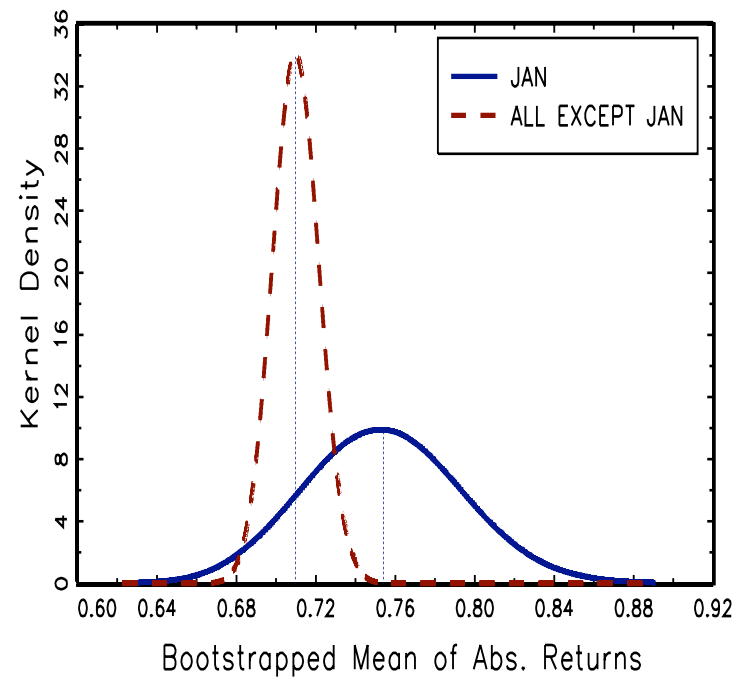

OCTOBER EXCLUDING 3 DAYS

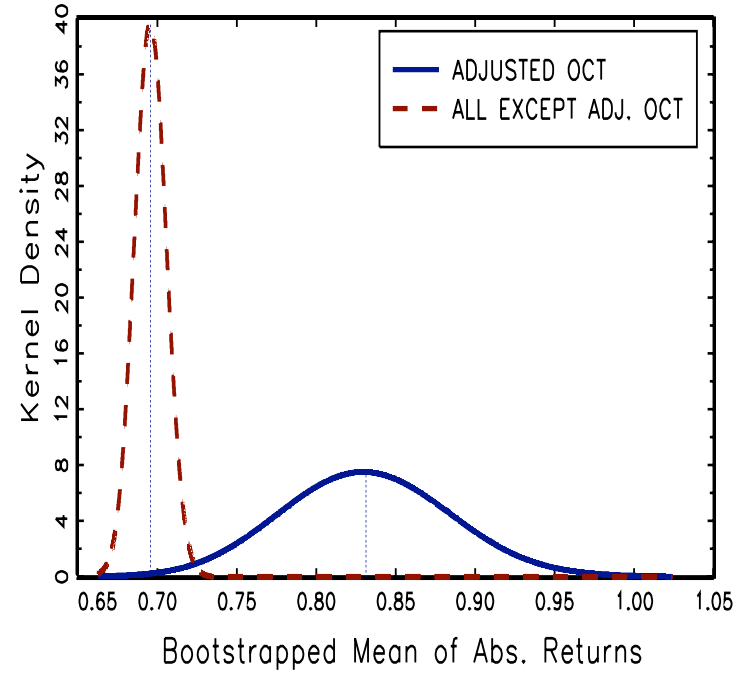

HOLIDAY

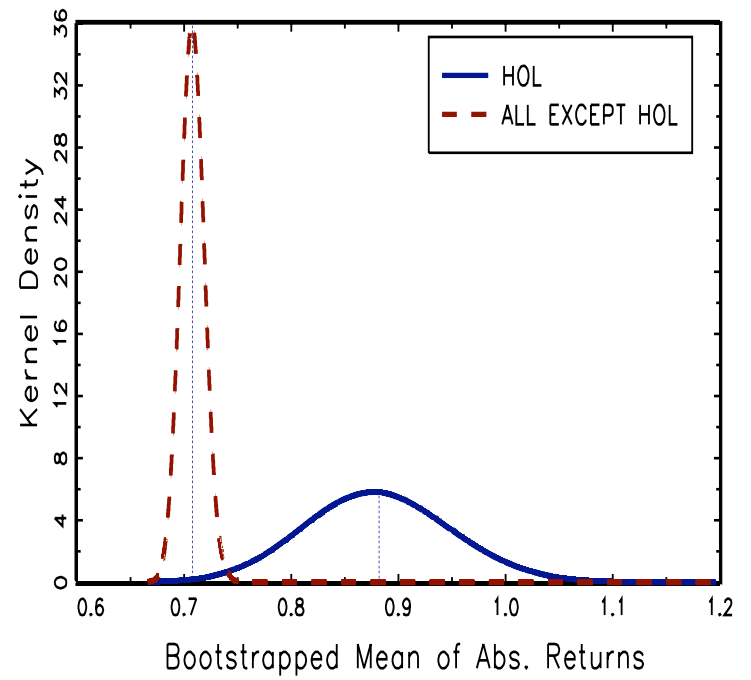

Figure 3: The October, January, and Holiday volatility effects. These are the kernel densities of the means of absolute returns generated by 10,000 bootstrap samples of the original sample. The vertical lines are drawn at the sample means. The adjusted sample for October excludes the three most volatile days of the sample: Monday, October 19, 1987 to Wednesday, October $21,1987$. 


\section{MONTH OF THE YEAR EFFECT}

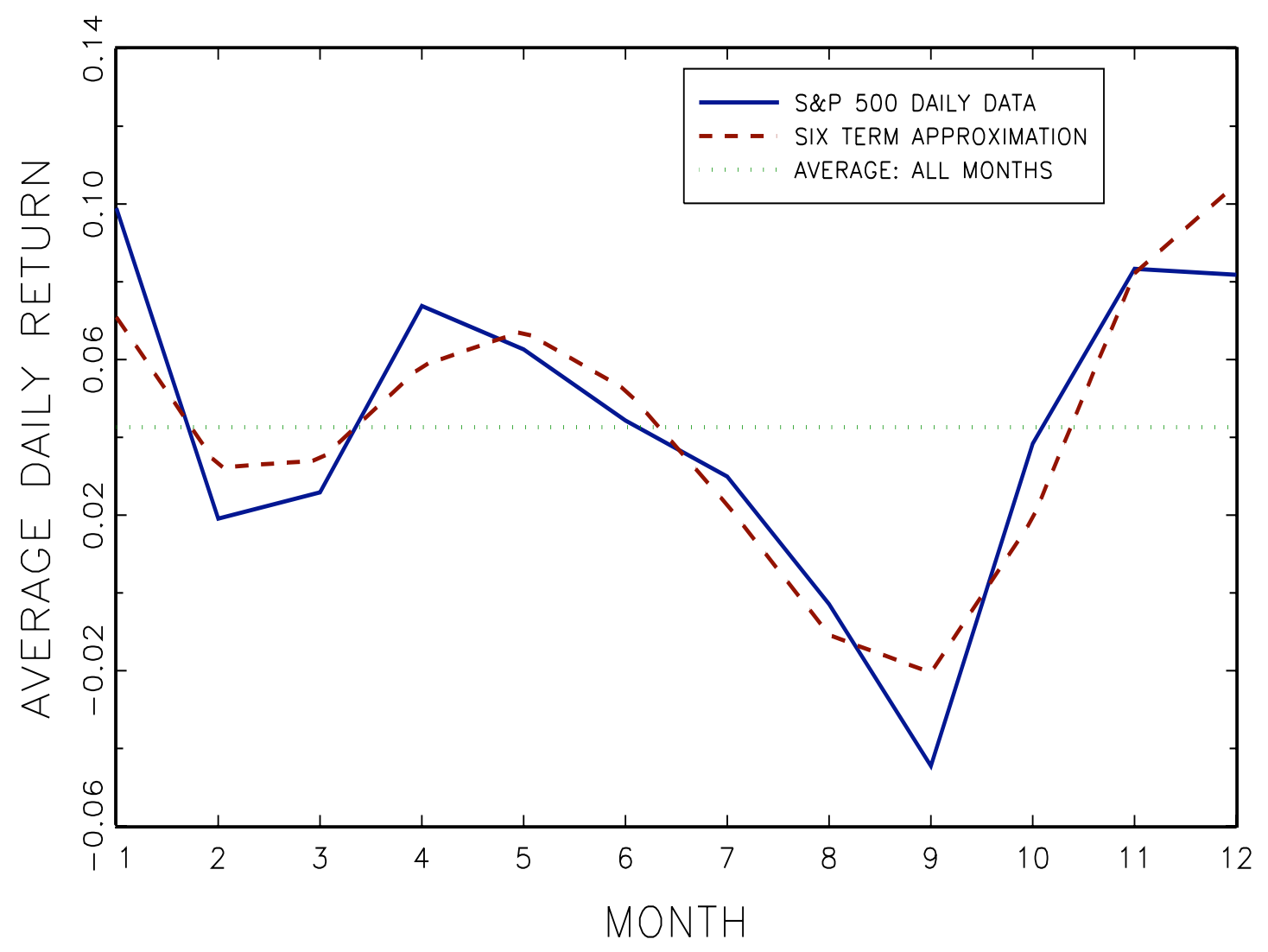

Figure 4: The Fourier approximation. This is the fitted six-term Fourier approximation to the average month of the year effect, which is obtained by running a simple OLS regression of the daily returns on the six Fourier terms, and then using the estimated coefficients. 
$\mathrm{SV}_{\mathrm{t}}$ MINUS PSV

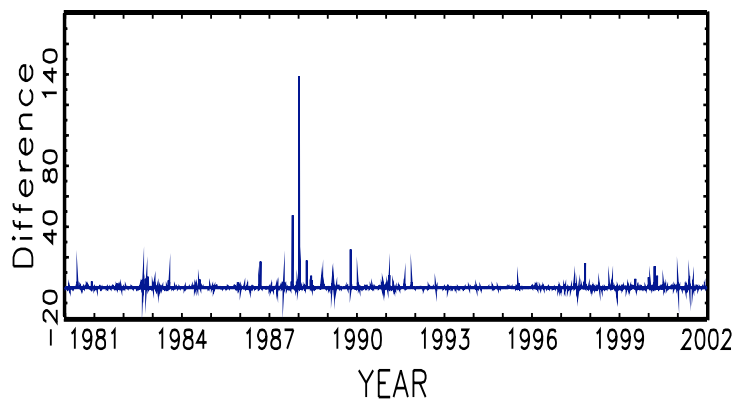

SV MINUS PSV

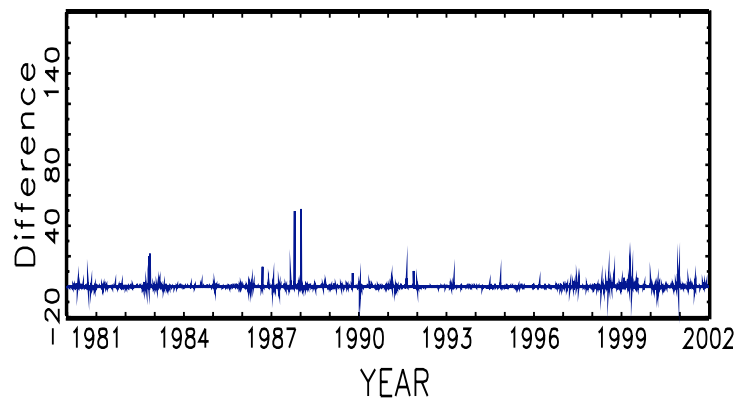

$S_{t}$ MINUS PSV

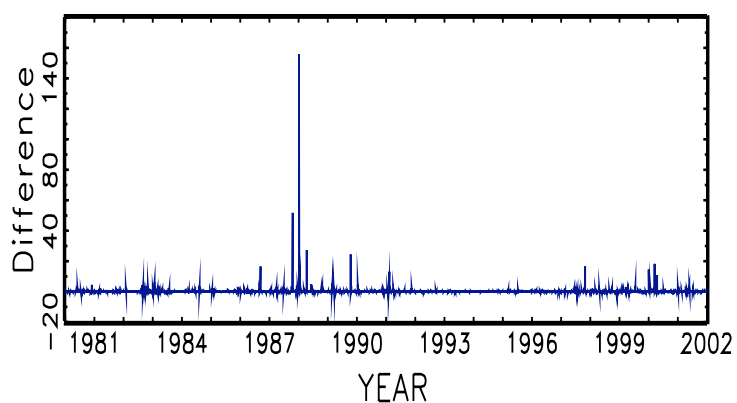

$S V_{t}$ MINUS PSVt

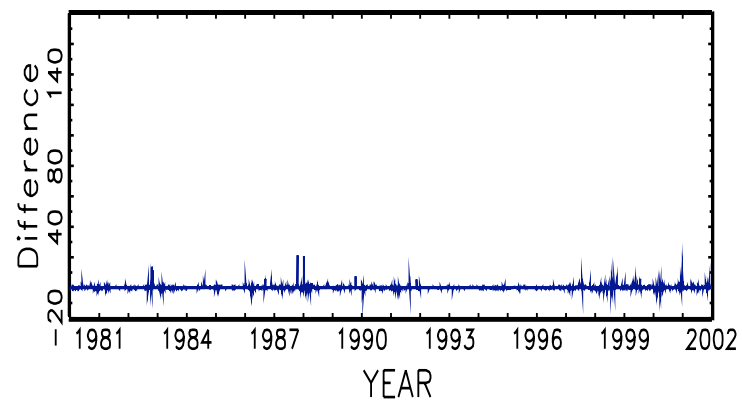

SV $V_{t}$ MINUS PSVt

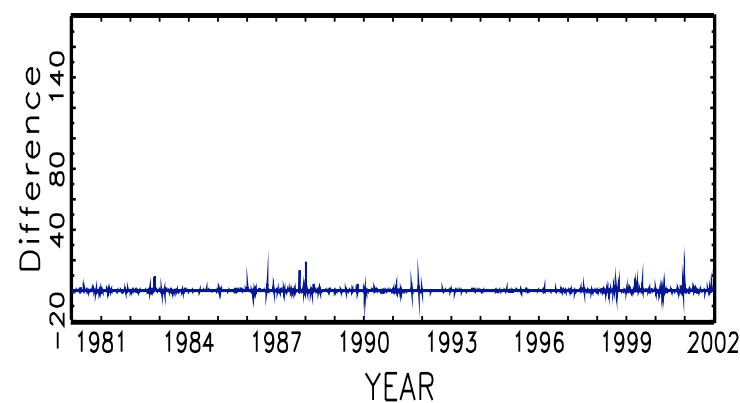

$S V_{t}$ MINUS PSVt

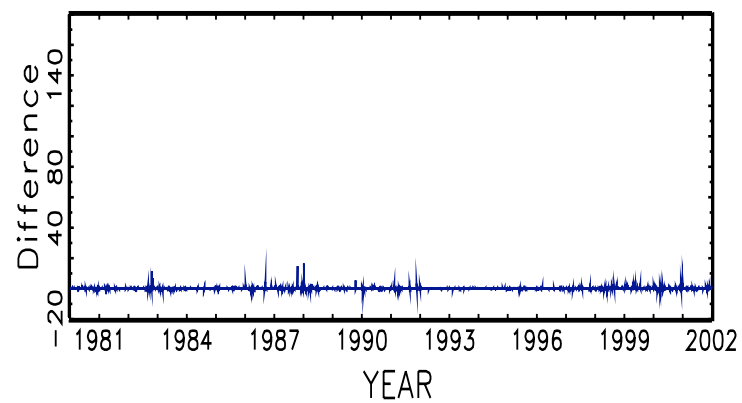

Figure 5: The predictive log-densities. This is the period-by-period difference in the predictive log-density of the $S V t$ model minus that of each of the PSV and PSVt specifications. 


\section{CUMULATIVE DIFFERENCE}

IN PREDICTIVE LOG-DENSITIES
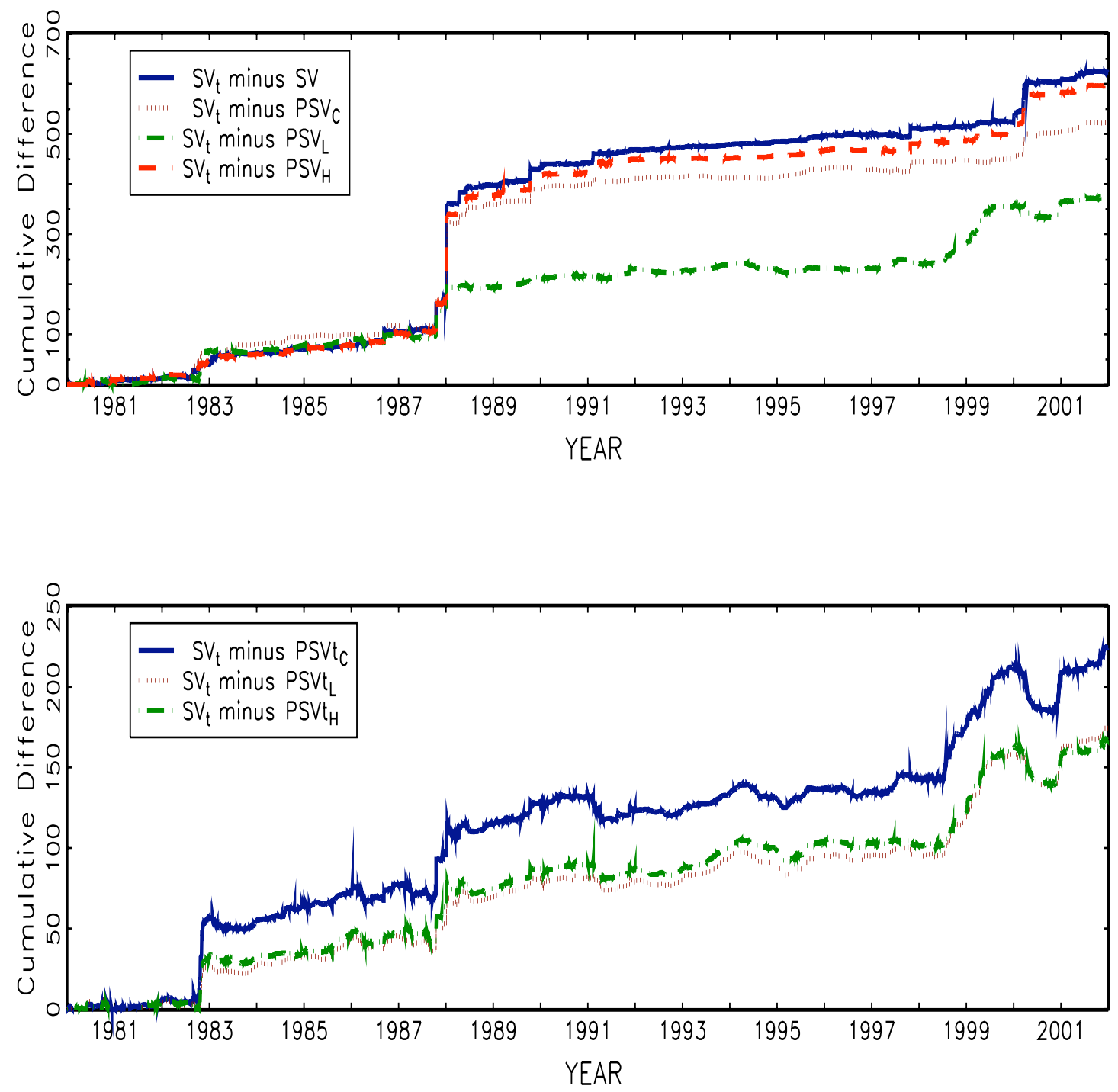

Figure 6: The cumulative log-densities. This is the cumulative period-by-period difference in the predictive log-density of the $S V t$ model minus that of each of the PSV and PSVt specifications. The final point is just the difference in the log-likelihoods. 
SV

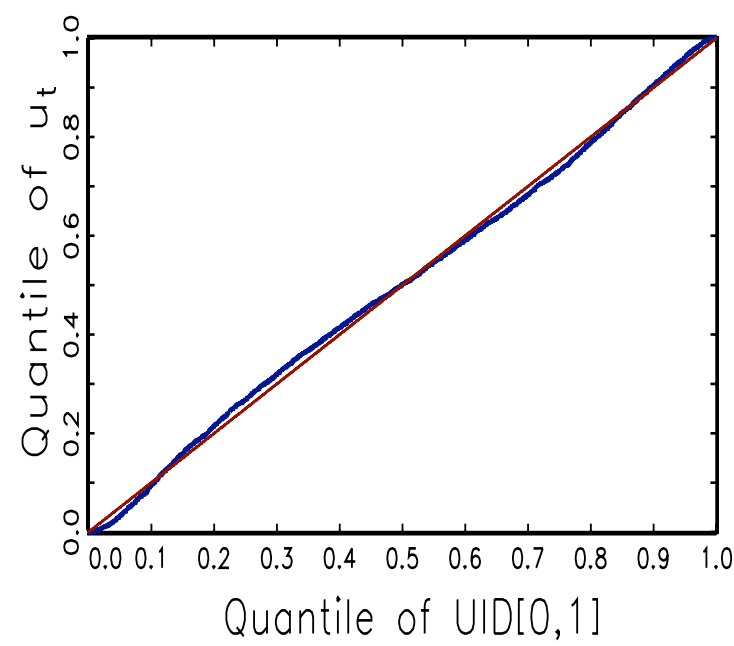

PSV

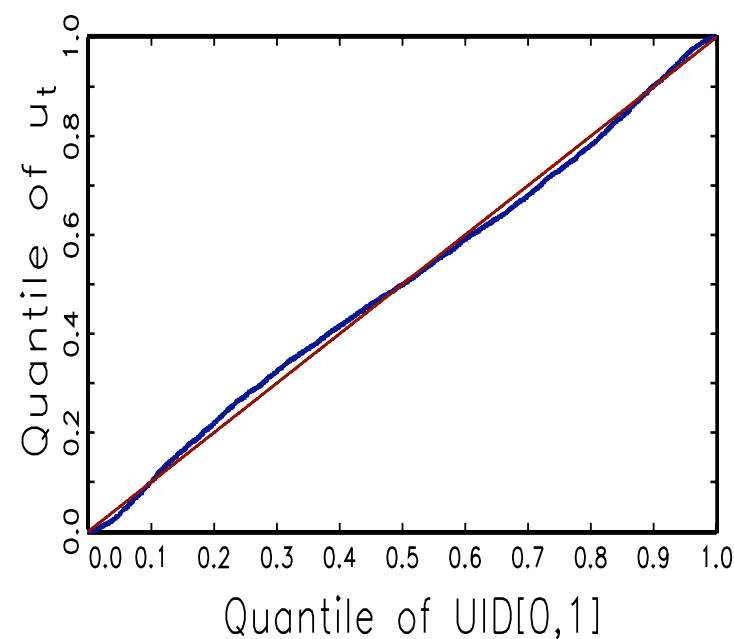

$P S V_{C}$

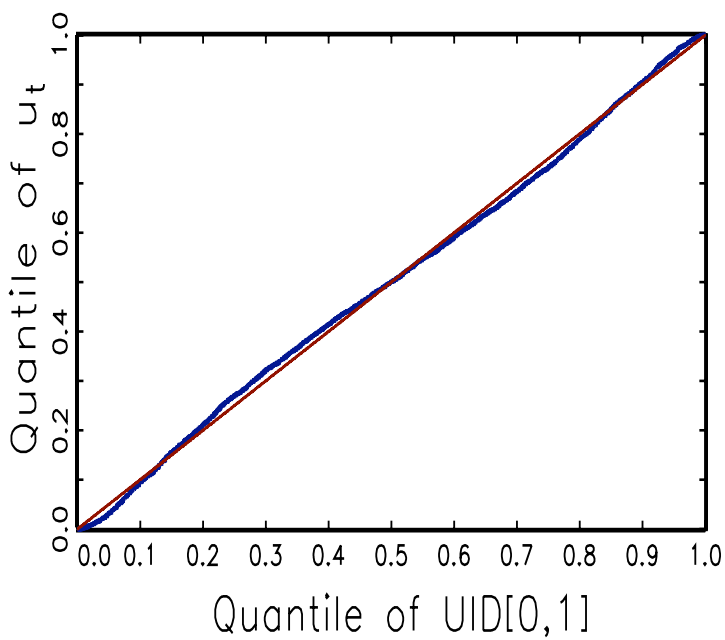

$P S V_{H}$

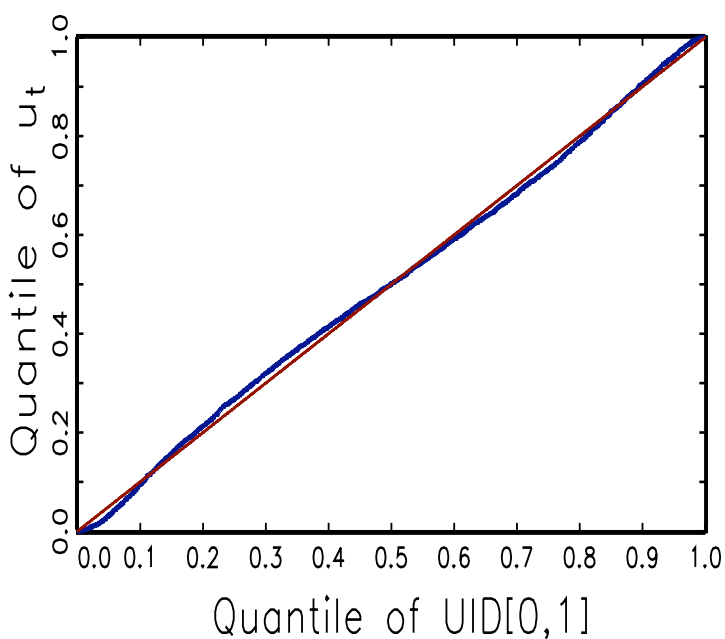

Figure 7: QQ plots for the four Gaussian $S V$ and $P S V$ models. 
SVt

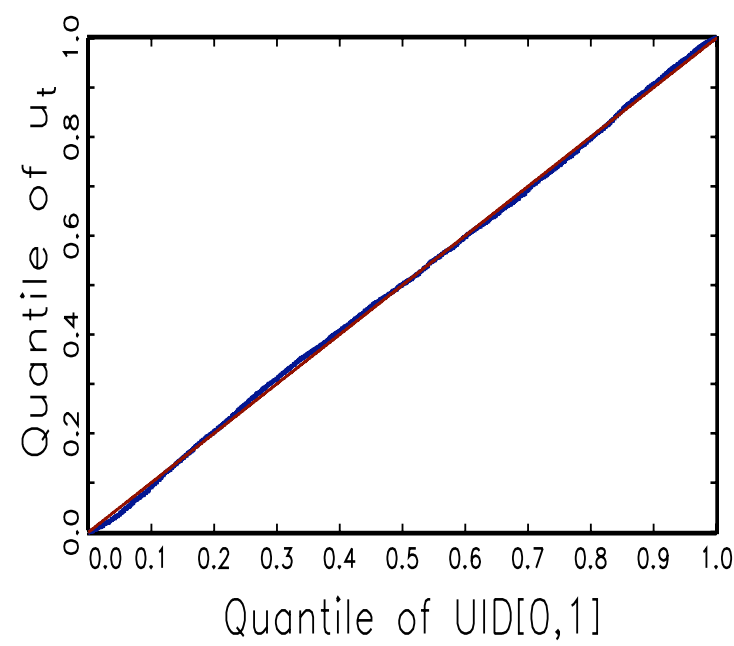

PSVtL

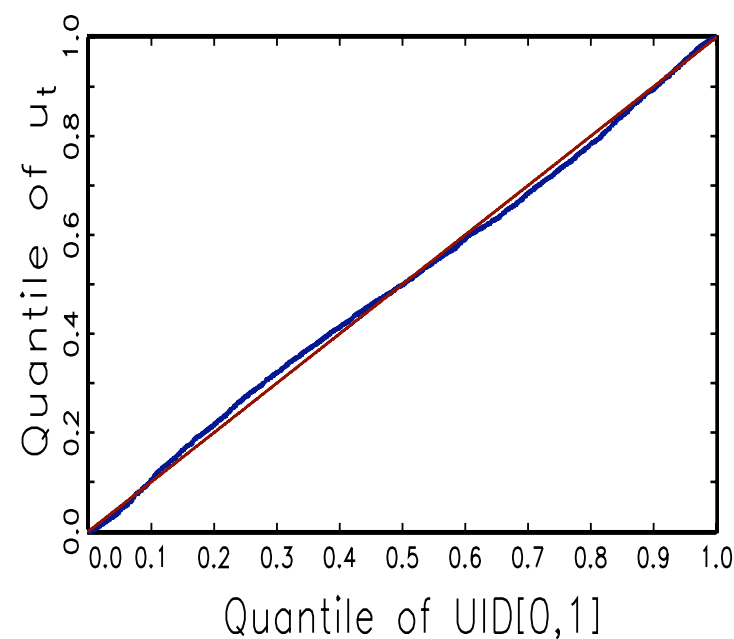

PSVtc

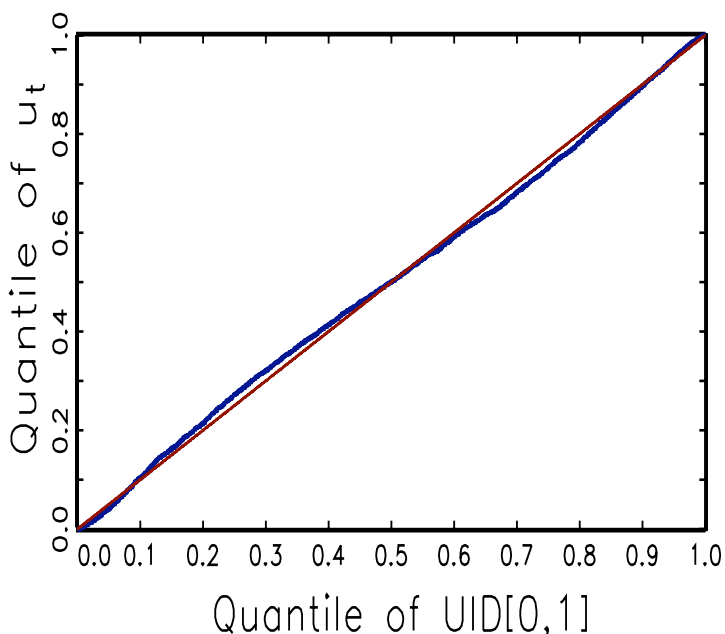

PSVt

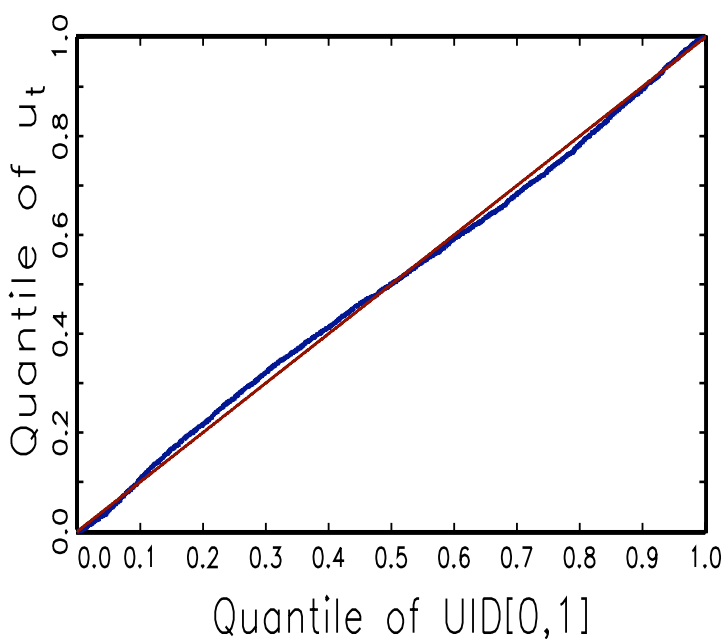

Figure 8: QQ plots for the four fat-tailed $S V t$ and PSVt models 


\section{References}

Admanti, A.R., and P. Pfeiderer, (1988). "A Theory of Intraday Patterns: Volume and Price Variability," Review of Financial Studies, 1, 3-40.

Admanti, A.R., and P. Pfeiderer, (1989). "Divide and Conquer: A Theory of Intraday and Day of the Week Mean Effects," Review of Financial Studies, 2, 189-223.

Andersen, T.G., and T. Bollerslev, (1997). "Intraday Periodicity and Volatility Persistence in Financial Markets," Journal of Empirical Finance, 4, 115-158.

Baillie, R.T., and T. Bollerslev, (1989). "The Message in Daily Exchange Rates: A Conditional Variance Tale," Journal of Business and Economic Statistics, 7, 297-305.

Balaban, E., A. Bayar, and O.B. Kan, (2001). "Stock returns, Seasonality and Asymmetric Conditional Volatility in World Equity Markets," Applied Economics Letters, 8, 263-268.

Beller, K., and J.R. Nofsinger, (1998). "On Stock Return Seasonality and Conditional Heteroscedasticity," Journal of Financial Research, 21, 229-246.

Beltratti, A., and C. Morana, (1999). "Computing Value at Risk with High Frequency Data" Journal of Empirical Finance, 6, 431-455.

Berkowitz, J., (2001). "Testing Density Forecasts with Applications to Risk Management," Journal of Business and Economic Statistics, 19, 465-74.

Berument, H., and H. Kuymaz, (2001). "The Day of the Week Effect on Stock Market Volatility," Journal of Economics and Finance, 25, 181-193.

Bollerslev, T., and E. Ghysels, (1996). "Periodic Autoregressive Conditional Heteroscedasticity," Journal of Business and Economic Statistics, 14, 139-151.

Bowman, K.O., and L.R. Shenton, (1975). "Omnibus Test Contours for Departures from Normality Based on $\sqrt{b_{1}}$ and $b_{2}$, " Biometrika, 62, 243-250.

Chib, S. (1995). "Marginal Likelihood from the Gibbs Output," Journal of the American Statistical Association, 90, 1313-1321

Chib, S., and I. Jeliazkov, (2001). "Marginal Likelihood from the Metropolis-Hastings Output," Journal of the American Statistical Association, 96, 270-281.

Chib, S., F. Nardari, and N. Shephard, (2002). "Markov Chain Monte Carlo Methods for Stochastic Volatility Models," Journal of Econometrics, 108, 281-316.

Flannery, M.J., and A.A. Protopapadakis, (1988). "From T-Bills to Common Stocks: Investigating the Generality of Intra-Week Return Seasonality," Journal of Finance, 33, 431-450. 
Fleming, J., and C. Kirby, (2003). "A Closer Look at the Relation between GARCH and Stochastic Autoregressive Volatility," Journal of Financial Econometrics, 1, 365-419.

Frances, P.H., R. Paap, (2000). "Modelling Day-of-the Week Seasonality in the S\&P 500 Index," Applied Financial Economics, 10, 483-488.

French, K.R., (1980). "Stock Returns and the Weekend Effect," Journal of Financial Economics, 8, 55-69.

French, K.R., and R. Roll, (1986). "Stock Return Variances: The Arrival of Information and the Reaction of Traders," Journal of Financial Economics, 17, 5-26.

Gallant, A.R., (1981). "On the Bias in Flexible Functional Forms and an Essentially Unbiased Form: The Fourier Flexible Form" Journal of Econometrics, 15, 211-245.

Gallant, A.R., P.E. Rossi, and G.E. Tauchen, (1992). "Stock Prices and Volume," The Review of Financial Studies, 5, 199-242.

Geweke, J., (1989). "Bayesian Inference in Econometric Models Using Monte Carlo Integration," Econometrica, 57, 1317-1339.

Geweke, J., (1992). "Evaluating the Accuracy of Sampling-Based Approaches to the Calculation of Posterior Moments." in Bayesian Statistics 4, eds. J.M. Bernardo, J. Berger, A.P. Dawid, and A.F.M. Smith, Oxford, U.K.: Oxford UP, 169-193.

Glosten, L.R., R. Jagannathan, and D.E. Runkle, (1993). "On the Relation between the Expected Value and the Volatility of the Nominal Excess Return on Stocks," Journal of Finance, 48, 1779-1801.

Hansen, B., (2003). "The Bootstrap" in Econometrics, Graduate lecture notes at the Economics Department, University of Winsconsin, 116-117.

Hansen, P.R., and A. Lunde, (2003). "Testing the Significance of Calendar Effects," Brown University: Working Paper 2003-03.

Haugen, R.A., and J. Lakonishok, (1988). The Incredible January Effect, Dow Jones-Irwin, Homewood Ill.

Hull, J., and A. White, (1987). "The Pricing of Options on Assets with Stochastic Volatilities," Journal of Finance, 42, 281-300.

Jacquier, E., N. Polson, and P. Rossi, (2002). "Bayesian Analysis of Stochastic Volatility Models," Journal of Business and Economic Statistics, 20, 69-87.

Johannes, M.S., R. Kumar, and N. Polson, (1999). "State Dependent Jump Models: How do U.S. Equity Markets Jump?" Manuscript, Columbia Business School. 
Johannes, M., and N. Polson, (2002). "MCMC Methods in Financial Econometrics" Handbook of Financial Econometrics, Ait-Sahalia Y. and Hansen L.P. eds., forthcoming.

Kass, R.E., and A.E. Raftery, (1995). "Bayes Factors," Journal of the American Statistical Association, 90, 773-795.

Keim, D.B., (1983). "Size Related Anomalies and Stock return Seasonality: Further Empirical Evidence," Journal of Financial Economics, 12, 13-32.

Keim, D.B., and R.F. Stambaugh, (1984). "A Further Investigation of the Weekend Effect in Stock Returns," Journal of Finance, 39, 819-835.

Kim, S., N. Shephard, and S. Chib, (1998). "Stochastic Volatility: Likelihood Inference and Comparison with ARCH models," Review of Economic Studies, 65, 361-393.

Lakonishok, J., and M. Levi, (1982). "Weekend Effects on Stock Returns," Journal of Finance, 37, 883-889.

Lakonishok, J., and E. Maberly, (1990). "The Weekend Effect: Trading Patterns of Individual and Institutional Investors," Journal of Finance, 40, 231-243.

Lakonishok, J., and S. Smidt, (1986). "Volume for Winners and Losers: Taxation and Other Motives for Stock Trading," Journal of Finance, 41, 951-974.

Lakonishok, J., and S. Smidt, (1988). "Are Seasonal Anomalies Real? A Ninety-Year Perspective," Review of Financial Studies, 1, 403-425.

Liesenfeld, R., and R.C. Jung, (2000). "Stochastic Volatility Models: Conditional Normality versus Heavy-Tailed Distributions," Journal of Applied Econometrics, 15, 137-160.

Penman, S.H., (1987). "The Distribution of Earnings News Over Time and Seasonalities in Aggregate Stock Returns," Journal of Financial Economics, 18, 199-228.

Pitt, M.K., and N. Shephard, (1999). "Filtering via Simulation Based on Auxiliary Particle Filters," Journal of the American Statistical Association, 94, 590-599.

Reinganum, M.R., (1983). "The Anomalous Stock Market Behaviour of Small Firms in January: Empirical Tests for Tax-Loss Selling Effects," Journal of Financial Economics, 12, 89-104.

Ritter, J.R., (1988). "The Buying and Selling Behaviour of Individual Investors at the Turn of the Year," Journal of Finance, 43, 701-717.

Roll, R., (1983). "Vas Ist das? The Turn-of-the Year Effect and the Return Premia of Small Firms," Journal of Portfolio Management, 9, 18-28. 
Rosenblatt, M., (1952). "Remarks on a Multivariate Transformation," Annals of Mathematical Statistics, 23, 470-2.

Sandmann, G., and S.J. Koopman, (1998). "Estimation of Stochastic Volatility Models via Monte Carlo Maximum Likelihood," Journal of Econometrics, 87, 271-301.

Sullivan, R., A. Timmermann, and H. White, (2001). "Dangers of Data Mining: The Case of Calendar Effects in Stock Returns," Journal of Econometrics, 105, 249-286.

\section{A APPENDIX A}

\section{A.1 One-Sided Hypothesis Testing using Bootstrapping ${ }^{17}$}

Suppose we want to test $H_{0}: \theta_{1}=\theta_{2}$ or equivalently $\theta_{1}-\theta_{2}=0$ against $H_{1}: \theta_{1}<\theta_{2}$ or equivalently $\theta_{1}-\theta_{2}<0$ at size $\alpha$. We construct the test statistic $T=\frac{\widehat{\theta}_{1}-\widehat{\theta}_{2}-0}{s\left(\widehat{\theta}_{1}-\widehat{\theta}_{2}-0\right)}=\frac{\widehat{\theta}_{1}-\widehat{\theta}_{2}}{s\left(\widehat{\theta}_{1}-\widehat{\theta}_{2}\right)}$ and reject in favour of $H_{1}$ if $T<c$. The standard error in testing for the difference between two sample means for unequal sample sizes, different population variances and independent groups is computed as $s\left(\widehat{\theta}_{1}-\widehat{\theta}_{2}\right)=\sqrt{\frac{\widehat{\sigma}_{1}^{2}}{T_{1}-1}+\frac{\widehat{\sigma}_{2}^{2}}{T_{2}-1}}$. The critical value $c$ is selected so that $\operatorname{Pr}(T<c)=a$ or $c=q_{\alpha}$, where $q_{\alpha}$ is the quantile of the empirical distribution of test statistic $T$ at the significance level $\alpha$. Since $q_{\alpha}$ is unknown, a bootstrap test replaces it with the bootstrap estimate $q_{\alpha}^{B}$ and the test rejects if $T<q_{\alpha}^{B}$. Similarly, if the alternative is $H_{1}: \theta_{1}>\theta_{2}$ or $\theta_{1}-\theta_{2}>0$, the bootstrap test rejects if $T>q_{1-\alpha}^{B}$.

Computationally, the critical value can be estimated from a bootstrap simulation by sorting the bootstrap $t$-statistics $T=\frac{\left(\widehat{\theta}_{1, b}^{*}-\widehat{\theta}_{2, b}^{*}\right)-\left(\widehat{\theta}_{1}-\widehat{\theta}_{2}\right)}{s\left(\widehat{\theta}_{1}^{*}-\widehat{\theta}_{2}^{*}\right)}$, where $\widehat{\theta}_{1, b}^{*}$ is the sample mean of $\theta_{1}$ in the $b$ 'th of a total of $B$ bootstrap samples. It is important to note that the bootstrap test statistic is centered at the estimate $\widehat{\theta}_{1}-\widehat{\theta}_{2}$, and the standard error $s\left(\widehat{\theta}_{1}^{*}-\widehat{\theta}_{2}^{*}\right)$ is calculated on the bootstrap samples as $s\left(\widehat{\theta}_{1}^{*}-\widehat{\theta}_{2}^{*}\right)=\sqrt{\operatorname{Var}\left(\widehat{\theta}_{1}^{*}-\widehat{\theta}_{2}^{*}\right)}$, where $\operatorname{Var}\left(\widehat{\theta}_{1}^{*}-\widehat{\theta}_{2}^{*}\right)=$ $\frac{1}{B} \sum_{b=1}^{B}\left\{\left(\widehat{\theta}_{1, b}^{*}-\widehat{\theta}_{2, b}^{*}\right)-\left(\overline{\hat{\theta}}_{1}^{*}-\overline{\hat{\theta}}_{2}^{*}\right)\right\}^{2}$, where $\overline{\boldsymbol{\theta}}_{1}^{*}$ is the average of the bootstrap means across all the $B$ bootstrap samples. Note that even though we generate the same number of bootstrap samples $B$ for both variables, it is the case that $\widehat{\theta}_{1}$ and $\widehat{\theta}_{2}$ (and hence $\widehat{\theta}_{1, b}^{*}-\widehat{\theta}_{2, b}^{*}$ ) are constructed using different original sample sizes $T$, but the same number of bootstrap samples $B$. We set $B=10,000$ bootstrap samples. These $t$-statistics are then sorted to find the estimated quantiles $q_{\alpha}^{B}$ or $q_{1-\alpha}^{B}$.

\footnotetext{
${ }^{17}$ For the details on hypothesis testing using bootstrapping see Hansen (2003).
} 


\section{B APPENDIX B: Sampling the Seasonal Level Volatil- ity Effects}

The parameters of all SV models examined in this paper are estimated using the Bayesian MCMC tools of Chib, Nardari and Shephard (2002), which build on the procedures developed by Kim, Shephard and Chib (1998). The algorithm constructs a Markov chain whose limiting distribution is the target posterior density of interest. Here, the Markov chain is a Gibbs sampler where all parameters are drawn sequentially from their full conditional posterior distribution. The Gibbs sampler is iterated a large number of times and the sampled draws, beyond a burn-in period, are treated as variates from the target distribution.

The periodic specifications require estimation of a high-dimensional parameter vector. For example, in the case of the PSVt model, the MCMC algorithm produces estimates of the posterior means of (i) the parameters of the return equation $\theta_{1}=\{\alpha, \rho\}$, (ii) the degrees of freedom parameter $\nu$ of the Student- $t$ distribution, (iii) the log-volatility parameters $\theta_{2}=$ $\left\{\mu, \beta_{1}, \phi, \sigma^{2}\right\}$, and (iv) the seasonal parameters $\theta_{3}=\{\gamma, \delta\}$ for the day of the week and month of the year effects in both the mean $\left\{\delta_{j}\right\}$ and the variance $\left\{\gamma_{j}\right\}, j \leq 11$. The key to estimating the high-dimensional SV models is the efficient sampling of the seasonal level effects in the conditional variance. This is done using a simple Gibbs step where the $\gamma=\left\{\gamma_{j}\right\}$ vector is drawn conditional on the log-variance vector $\left\{h_{t}\right\}$ using a precision-weighted average of prior information and the conditional likelihood. Despite its simplicity, this method is numerically superior to the sampling of $\gamma$ in the same block as the log-volatility parameters $\theta_{2}$.

Specifically, the estimation of $\theta_{2}$ involves a numerical optimization step, which generates a proposal that is accepted according to the Metropolis-Hastings algorithm. Sampling $\gamma$ in the same block as $\theta_{2}$ requires high-dimensional optimization, which in turn may cause problems such as slowing the algorithm considerably, returning a non-positive definite numerical Hessian matrix, or producing an unacceptably high $\mathrm{M}-\mathrm{H}$ rejection rate. The results indicate that the simple Gibbs step is highly efficient in that the posterior mean estimates of $\gamma$ have very low NSE and RNI values.

\section{B.1 A brief sketch of the MCMC algorithm}

1. Initialize $\theta, s, \lambda, \nu$ and transform the data into $y_{t}^{*}=\log \left(\frac{\omega_{t}}{\lambda_{t}^{-1}}+c\right), c=0.001$ to put the model in state-space form. The "offset" constant $c$ eliminates the inlier problem.

2. Sample all the log-volatility parameters from their full conditional posterior density: $\theta_{2} \mid y^{*}, s, \gamma$. This posterior is not available analytically. We use the Kalman filter to compute the log-likelihood of transformed data $y_{t}^{*}$ as a function of $\theta$ (conditional on $s_{t}$ ) and then optimize this conditional log-posterior. We generate a proposal from a $t$ distribution $t(m, V, \xi)$ where $m$ is the mode, $V$ is the inverse of the negative Hessian and $\xi$ a tuning parameter. The proposal is then accepted according to the Metropolis-Hastings algorithm. The optimization step makes this an independence chain $\mathrm{M}-\mathrm{H}$ algorithm and 
goes a long way in reducing the autocorrelation in the draws of the MCMC chain. For more details on the M-H algorithm see Chib and Greenberg (1995).

3. Sample the seasonal coefficients in the log-variance equation from their full conditional posterior $\gamma \mid y^{*}, D, h, s$ using the Gibbs step detailed below separately.

4. Sample the log-volatility vector $\left\{h_{t}\right\}$ in one block from the full conditional posterior distribution: $h \mid y^{*}, s, \theta$. This step uses the de Jong and Shephard (1995) simulation smoother which is an algorithm designed for efficient sampling of the state vector in a state-space model.

5. Sample the degrees of freedom parameter of the conditional distribution from the full conditional density: $\nu \mid y, h, \theta$. Again, we optimize the conditional log-posterior with respect to $\nu$ and then use the mode and a scaled inverse of the negative Hessian to generate a proposal that is accepted according to the Metropolis-Hastings algorithm. This independence chain $\mathrm{M}-\mathrm{H}$ algorithm is also crucial in contributing to low Relative Numerical Inefficiencies for the parameters of interest.

6. Sample $\lambda \mid y, h, \theta$ directly from its posterior:

$$
\lambda_{t} \mid y_{t}, h_{t}, \theta \sim \operatorname{Gamma}\left(\frac{\nu+1}{2}, \frac{2}{\nu+\omega_{t}^{2} / v_{t}^{2}}\right)
$$

7. Sample all the conditional mean coefficients (including the seasonal coefficients in the mean) $\theta_{1}, \delta \mid y, D, h, \lambda$ simply using a precision-weighted average of a set of normal priors and the normal conditional likelihood. Then update the transformed data $y_{t}^{*}=$ $\log \left(\frac{\omega_{t}^{2}}{\lambda_{t}^{-1}}+c\right), c=0.001$.

8. Finally, sample the mixture indicator variable $s \mid y^{*}, h, \theta$ directly from its posterior:

$$
\operatorname{Pr}\left(s_{t} \mid y_{t}^{*}, h_{t}\right) \propto \operatorname{Pr}\left(s_{t}\right) \phi\left(y_{t}^{*} \mid h_{t}+\gamma^{\prime} D_{t}+m_{s t}, v_{s t}^{2}\right), \quad t \leq T
$$

9. Go to step 2 and iterate.

\section{B.2 The Gibbs step}

Consider the state-space representation of the periodic SV model

$$
\begin{gathered}
y_{t}^{*}=\gamma^{\prime} D_{t}+h_{t}+z_{t}, \quad z_{t} \mid s_{t} \sim N\left(m_{s t}, v_{s t}^{2}\right) \\
h_{t}=\mu+\beta_{1} y_{t-1}+\phi\left(h_{t-1}-\mu\right)+\sigma \eta_{t}, \quad \eta_{t} \sim N I D(0,1)
\end{gathered}
$$




$$
\gamma^{\prime} D_{t}=\sum_{j=1}^{11} \gamma_{j} D_{j, t}
$$

We wish to sample the coefficients of the level volatility effects $\gamma=\left\{\gamma_{j}\right\}, j \leq 11$ from $\gamma \mid y^{*}, D, h, s$ using a precision-weighted average of the prior and the conditional likelihood. Define the vector of priors $\gamma \sim N\left(\gamma_{0}, \Gamma_{0}^{-1}\right)$, where $\gamma_{0}=[0, \ldots, 0]^{\prime} \in \Re^{11}$ and $\Gamma_{0}^{-1}=I_{11}$. Consider the model equation

$$
y_{t}^{*}=\gamma^{\prime} D_{t}+h_{t}+z_{t}, \quad z_{t} \mid s_{t} \sim N\left(m_{s t}, v_{s t}\right)
$$

and rename

$$
\widehat{y_{t}}=\frac{y_{t}^{*}-h_{t}-m_{s t}}{v_{s t}}, \quad \widehat{D}_{j, t}=\frac{D_{j, t}}{v_{s t}}
$$

Then, the posterior mean estimates of the $\gamma$ vector are simply given by

$$
\begin{gathered}
\widehat{\gamma}=V_{\gamma}\left[\Gamma_{0} \gamma_{0}+\widehat{D}^{\prime} \widehat{y}\right] \in \Re^{11} \\
V_{\gamma}=\left[\Gamma_{0}+\widehat{D}^{\prime} \widehat{D}\right]^{-1} \in \Re^{11 \times 11}
\end{gathered}
$$




\section{List of other working papers:}

\section{4}

1. Xiaohong Chen, Yanqin Fan and Andrew Patton, Simple Tests for Models of Dependence Between Multiple Financial Time Series, with Applications to U.S. Equity Returns and Exchange Rates, WP04-19

2. Valentina Corradi and Walter Distaso, Testing for One-Factor Models versus Stochastic Volatility Models, WP04-18

3. Valentina Corradi and Walter Distaso, Estimating and Testing Sochastic Volatility Models using Realized Measures, WP04-17

4. Valentina Corradi and Norman Swanson, Predictive Density Accuracy Tests, WP04-16

5. Roel Oomen, Properties of Bias Corrected Realized Variance Under Alternative Sampling Schemes, WP04-15

6. Roel Oomen, Properties of Realized Variance for a Pure Jump Process: Calendar Time Sampling versus Business Time Sampling, WP04-14

7. Richard Clarida, Lucio Sarno, Mark Taylor and Giorgio Valente, The Role of Asymmetries and Regime Shifts in the Term Structure of Interest Rates, WP04-13

8. Lucio Sarno, Daniel Thornton and Giorgio Valente, Federal Funds Rate Prediction, WP04-12

9. Lucio Sarno and Giorgio Valente, Modeling and Forecasting Stock Returns: Exploiting the Futures Market, Regime Shifts and International Spillovers, WP04-11

10. Lucio Sarno and Giorgio Valente, Empirical Exchange Rate Models and Currency Risk: Some Evidence from Density Forecasts, WP04-10

11. Ilias Tsiakas, Periodic Stochastic Volatility and Fat Tails, WP04-09

12. Ilias Tsiakas, Is Seasonal Heteroscedasticity Real? An International Perspective, WP04-08

13. Damin Challet, Andrea De Martino, Matteo Marsili and Isaac Castillo, Minority games with finite score memory, WP04-07

14. Basel Awartani, Valentina Corradi and Walter Distaso, Testing and Modelling Market Microstructure Effects with an Application to the Dow Jones Industrial Average, WP04-06

15. Andrew Patton and Allan Timmermann, Properties of Optimal Forecasts under Asymmetric Loss and Nonlinearity, WP04-05

16. Andrew Patton, Modelling Asymmetric Exchange Rate Dependence, WP04-04

17. Alessio Sancetta, Decoupling and Convergence to Independence with Applications to Functional Limit Theorems, WP04-03

18. Alessio Sancetta, Copula Based Monte Carlo Integration in Financial Problems, WP04-02

19. Abhay Abhayankar, Lucio Sarno and Giorgio Valente, Exchange Rates and Fundamentals: Evidence on the Economic Value of Predictability, WP04-01

\section{2}

1. Paolo Zaffaroni, Gaussian inference on Certain Long-Range Dependent Volatility Models, WP02-12

2. Paolo Zaffaroni, Aggregation and Memory of Models of Changing Volatility, WP02-11

3. Jerry Coakley, Ana-Maria Fuertes and Andrew Wood, Reinterpreting the Real Exchange Rate - Yield Diffential Nexus, WP02-10

4. Gordon Gemmill and Dylan Thomas, Noise Training, Costly Arbitrage and Asset Prices: evidence from closed-end funds, WP02-09

5. Gordon Gemmill, Testing Merton's Model for Credit Spreads on Zero-Coupon Bonds, WP0208

6. George Christodoulakis and Steve Satchell, On th Evolution of Global Style Factors in the MSCI Universe of Assets, WP02-07

7. George Christodoulakis, Sharp Style Analysis in the MSCI Sector Portfolios: A Monte Caro Integration Approach, WP02-06 
8. George Christodoulakis, Generating Composite Volatility Forecasts with Random Factor Betas, WP02-05

9. Claudia Riveiro and Nick Webber, Valuing Path Dependent Options in the Variance-Gamma Model by Monte Carlo with a Gamma Bridge, WP02-04

10. Christian Pedersen and Soosung Hwang, On Empirical Risk Measurement with Asymmetric Returns Data, WP02-03

11. Roy Batchelor and Ismail Orgakcioglu, Event-related GARCH: the impact of stock dividends in Turkey, WP02-02

12. George Albanis and Roy Batchelor, Combining Heterogeneous Classifiers for Stock Selection, WP02-01

\section{1}

1. Soosung Hwang and Steve Satchell, GARCH Model with Cross-sectional Volatility; GARCHX Models, WP01-16

2. Soosung Hwang and Steve Satchell, Tracking Error: Ex-Ante versus Ex-Post Measures, WP01-15

3. Soosung Hwang and Steve Satchell, The Asset Allocation Decision in a Loss Aversion World, WP01-14

4. Soosung Hwang and Mark Salmon, An Analysis of Performance Measures Using Copulae, WP01-13

5. Soosung Hwang and Mark Salmon, A New Measure of Herding and Empirical Evidence, WP01-12

6. Richard Lewin and Steve Satchell, The Derivation of New Model of Equity Duration, WP0111

7. Massimiliano Marcellino and Mark Salmon, Robust Decision Theory and the Lucas Critique, WP01-10

8. Jerry Coakley, Ana-Maria Fuertes and Maria-Teresa Perez, Numerical Issues in Threshold Autoregressive Modelling of Time Series, WP01-09

9. Jerry Coakley, Ana-Maria Fuertes and Ron Smith, Small Sample Properties of Panel Timeseries Estimators with I(1) Errors, WP01-08

10. Jerry Coakley and Ana-Maria Fuertes, The Felsdtein-Horioka Puzzle is Not as Bad as You Think, WP01-07

11. Jerry Coakley and Ana-Maria Fuertes, Rethinking the Forward Premium Puzzle in a Nonlinear Framework, WP01-06

12. George Christodoulakis, Co-Volatility and Correlation Clustering: A Multivariate Correlated ARCH Framework, WP01-05

13. Frank Critchley, Paul Marriott and Mark Salmon, On Preferred Point Geometry in Statistics, WP01-04

14. Eric Bouyé and Nicolas Gaussel and Mark Salmon, Investigating Dynamic Dependence Using Copulae, WP01-03

15. Eric Bouyé, Multivariate Extremes at Work for Portfolio Risk Measurement, WP01-02

16. Erick Bouyé, Vado Durrleman, Ashkan Nikeghbali, Gael Riboulet and Thierry Roncalli, Copulas: an Open Field for Risk Management, WP01-01

\section{0}

1. Soosung Hwang and Steve Satchell, Valuing Information Using Utility Functions, WP00-06

2. Soosung Hwang, Properties of Cross-sectional Volatility, WP00-05

3. Soosung Hwang and Steve Satchell, Calculating the Miss-specification in Beta from Using a Proxy for the Market Portfolio, WP00-04

4. Laun Middleton and Stephen Satchell, Deriving the APT when the Number of Factors is Unknown, WP00-03

5. George A. Christodoulakis and Steve Satchell, Evolving Systems of Financial Returns: AutoRegressive Conditional Beta, WP00-02

6. Christian S. Pedersen and Stephen Satchell, Evaluating the Performance of Nearest Neighbour Algorithms when Forecasting US Industry Returns, WP00-01 
1. Yin-Wong Cheung, Menzie Chinn and Ian Marsh, How do UK-Based Foreign Exchange Dealers Think Their Market Operates?, WP99-21

2. Soosung Hwang, John Knight and Stephen Satchell, Forecasting Volatility using LINEX Loss Functions, WP99-20

3. Soosung Hwang and Steve Satchell, Improved Testing for the Efficiency of Asset Pricing Theories in Linear Factor Models, WP99-19

4. Soosung Hwang and Stephen Satchell, The Disappearance of Style in the US Equity Market, WP99-18

5. Soosung Hwang and Stephen Satchell, Modelling Emerging Market Risk Premia Using Higher Moments, WP99-17

6. Soosung Hwang and Stephen Satchell, Market Risk and the Concept of Fundamental Volatility: Measuring Volatility Across Asset and Derivative Markets and Testing for the Impact of Derivatives Markets on Financial Markets, WP99-16

7. Soosung Hwang, The Effects of Systematic Sampling and Temporal Aggregation on Discrete Time Long Memory Processes and their Finite Sample Properties, WP99-15

8. Ronald MacDonald and Ian Marsh, Currency Spillovers and Tri-Polarity: a Simultaneous Model of the US Dollar, German Mark and Japanese Yen, WP99-14

9. Robert Hillman, Forecasting Inflation with a Non-linear Output Gap Model, WP99-13

10. Robert Hillman and Mark Salmon, From Market Micro-structure to Macro Fundamentals: is there Predictability in the Dollar-Deutsche Mark Exchange Rate?, WP99-12

11. Renzo Avesani, Giampiero Gallo and Mark Salmon, On the Evolution of Credibility and Flexible Exchange Rate Target Zones, WP99-11

12. Paul Marriott and Mark Salmon, An Introduction to Differential Geometry in Econometrics, WP99-10

13. Mark Dixon, Anthony Ledford and Paul Marriott, Finite Sample Inference for Extreme Value Distributions, WP99-09

14. Ian Marsh and David Power, A Panel-Based Investigation into the Relationship Between Stock Prices and Dividends, WP99-08

15. Ian Marsh, An Analysis of the Performance of European Foreign Exchange Forecasters, WP99-07

16. Frank Critchley, Paul Marriott and Mark Salmon, An Elementary Account of Amari's Expected Geometry, WP99-06

17. Demos Tambakis and Anne-Sophie Van Royen, Bootstrap Predictability of Daily Exchange Rates in ARMA Models, WP99-05

18. Christopher Neely and Paul Weller, Technical Analysis and Central Bank Intervention, WP9904

19. Christopher Neely and Paul Weller, Predictability in International Asset Returns: A Reexamination, WP99-03

20. Christopher Neely and Paul Weller, Intraday Technical Trading in the Foreign Exchange Market, WP99-02

21. Anthony Hall, Soosung Hwang and Stephen Satchell, Using Bayesian Variable Selection Methods to Choose Style Factors in Global Stock Return Models, WP99-01

\section{8}

1. Soosung Hwang and Stephen Satchell, Implied Volatility Forecasting: A Compaison of Different Procedures Including Fractionally Integrated Models with Applications to UK Equity Options, WP98-05

2. Roy Batchelor and David Peel, Rationality Testing under Asymmetric Loss, WP98-04

3. Roy Batchelor, Forecasting T-Bill Yields: Accuracy versus Profitability, WP98-03

4. Adam Kurpiel and Thierry Roncalli, Option Hedging with Stochastic Volatility, WP98-02

5. Adam Kurpiel and Thierry Roncalli, Hopscotch Methods for Two State Financial Models, WP98-01 